WSRC-TR-2004-00555

Key Words:

Radionuclides

NCRP

Performance Assessment

Retention: Permanent

\title{
ATMOSPHERIC PATHWAY SCREENING ANALYSIS FOR SALTSTONE DISPOSAL FACILITY VAULT 4
}

\author{
Kimberly P. Crapse \\ James R. Cook
}

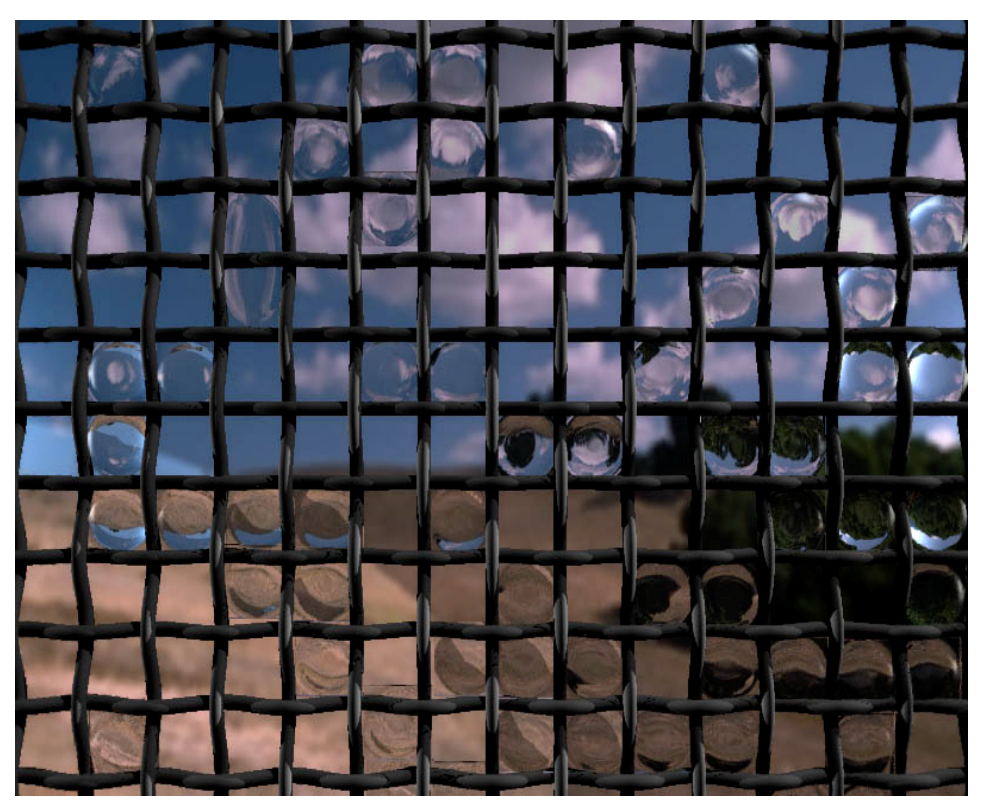

NOVEMBER 9, 2004

Westinghouse Savannah River Company Savannah River Site Aiken, SC 29808

Prepared for the U.S. Department of Energy Under Contract Number DE-AC09-96SR18500

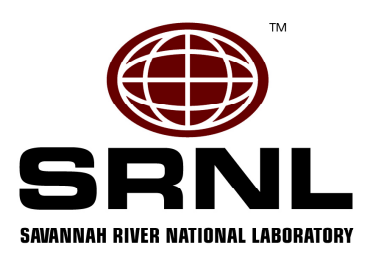


This document was prepared in conjunction with work accomplished under Contract No. DE-AC09-96SR18500 with the U. S. Department of Energy.

\section{DISCLAIMER}

This report was prepared as an account of work sponsored by an agency of the United States Government. Neither the United States Government nor any agency thereof, nor any of their employees, makes any warranty, express or implied, or assumes any legal liability or responsibility for the accuracy, completeness, or usefulness of any information, apparatus, product or process disclosed, or represents that its use would not infringe privately owned rights. Reference herein to any specific commercial product, process or service by trade name, trademark, manufacturer, or otherwise does not necessarily constitute or imply its endorsement, recommendation, or favoring by the United States Government or any agency thereof. The views and opinions of authors expressed herein do not necessarily state or reflect those of the United States Government or any agency thereof.

This report has been reproduced directly from the best available copy.

Available for sale to the public, in paper, from: U.S. Department of Commerce, National Technical Information Service, 5285 Port Royal Road, Springfield, VA 22161, phone: (800) 553-6847, fax: (703) 605-6900

email: orders@ntis.fedworld.gov

online ordering: http://www.ntis.gov/help/index.asp

Available electronically at http://www.osti.gov/bridge

Available for a processing fee to U.S. Department of Energy and its contractors, in paper, from: U.S. Department of Energy, Office of Scientific and Technical Information, P.O. Box 62, Oak Ridge, TN 37831-0062,

phone: (865)576-8401,

fax: (865)576-5728

email: $\underline{\text { reports@ adonis.osti.gov }}$ 
WSRC-TR-2004-00555

Key Words:

Radionuclides

NCRP

Performance Assessment

Retention: Permanent

\title{
ATMOSPHERIC PATHWAY SCREENING ANALYSIS FOR SALTSTONE DISPOSAL FACILITY VAULT 4
}

\author{
Kimberly P. Crapse \\ James R. Cook
}

NOVEMBER 9, 2004

Westinghouse Savannah River Company

Savannah River Site

Aiken, SC 29808

Prepared for the U.S. Department of Energy Under

Contract Number DE-AC09-96SR18500

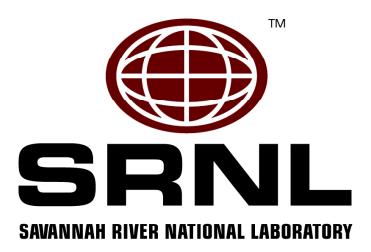




\section{EXECUTIVE SUMMARY}

A sequential screening process using a methodology developed by the National Council on Radiation Protection and Measurements, professional judgement and process knowledge has been used to produce a list of radionuclides requiring detailed analysis to derive disposal limits for the Saltstone Disposal Facility based on the atmospheric pathway.

\section{DISCUSSION}

The National Council on Radiation Protection and Measurements (NCRP) has published a report that described a methodology to screen out, or remove from further consideration, radionuclides for detailed analysis in a performance assessment (NCRP, 1996). The NCRP provides a screening methodology, which uses some conservative assumptions, a few facility-specific parameters and an estimated inventory to produce a dose estimate for each radionuclide. If the estimated dose exceeds the dose criteria, then that radionuclide must undergo further analysis.

This process was implemented for the Saltstone Disposal Facility by conservatively assuming an inventory of 10,000,000 curies for each radionuclide and a dose criteria of $0.1 \mathrm{mrem} / \mathrm{year}$. When the process was applied, 13 of the 826 radionuclides considered were removed from further consideration - Ar-37, Ar-39, At-215, At-216, Fr-219, Hf-174, Kr-81m, Ne-19, Po-211, Po-212, Po-213, Po-214 and Rn-218. The screening factors and doses are shown in Table 1.

In order to further reduce the number of radionuclides to be considered in the detailed analysis, some fundamental principles of physics and chemistry were applied. The performance assessment only considers times after final facility closure. Once the disposal vaults are filled and capped, there are only two possible ways for radionuclides to be released to the atmosphere. One is by particulates produced by intrusion, which will be considered separately in the performance assessment, and the other is by release as a gas. The list of elements comprising the remaining 813 radionuclides was examined to identify those which have the potential to form a vapor phase in the disposal vault. This produced the following elements: Ar, As, At, Br, C, Cl, F, Ge, H, Hg, I, $\mathrm{Kr}, \mathrm{N}, \mathrm{O}, \mathrm{P}, \mathrm{S}, \mathrm{Sb}, \mathrm{Se}, \mathrm{Sn}$ and $\mathrm{Xe}$. These elements have a total of 137 individual radionuclides. Radon was not considered further because it is treated separately in the performance assessment process (USDOE 1999).

Three Saltstone inventory estimate sources were used to develop a list of radionuclides thought to be in the feed to Saltstone (MMES, 1992; Cook et al., 2002; Chandler, 2004). Of the 137 radionuclides that could exist in the gas phase, 9 are thought to be in potential Saltstone feed: C14, Cl-36, H-3, I-129, Sb-125, Sb-126, Se-79, Sn-121m and Sn-126. A detailed analysis of the atmospheric pathway for these radionuclides will be performed.

Trigger values were calculated from the screening results for the remaining 142 radionuclides using the methodology developed for E-Area (Cook, 2004). If any of the 128 radionuclides does appear in the Saltstone feed, the quantity can be compared to the Trigger Value. If the estimated vault inventory will be less than the Trigger Value, then no further analysis is needed. If the estimated inventory exceeds the Trigger Value, a Special Analysis will be required before that feed can be accepted for disposal. The Trigger Values are given in Table 2.

\section{CONCLUSIONS}

Nine radionuclides have been determined to require detailed analysis for the atmospheric pathway. Trigger values have been developed for other radionuclides of potential interest to the atmospheric pathway. 
Table 1. Results of the Screening Analysis

\begin{tabular}{|c|c|c|c|}
\hline Radionuclide & $\begin{array}{c}\text { Level I } \\
\text { Screening Factor } \\
\left(\mathrm{Sv} / \mathrm{Bq}-\mathrm{m}^{-3}\right) \\
\end{array}$ & $\begin{array}{c}\text { Level I } \\
\text { Screening Factor } \\
\left(\mathrm{mrem} / \mathrm{Ci}-\mathrm{m}^{-3}\right) \\
\end{array}$ & $\begin{array}{l}\text { Screening } \\
\text { Dose } \\
\text { mrem/yr } \\
\end{array}$ \\
\hline Ac-223 & $1.6 \mathrm{E}-08$ & $5.9 \mathrm{E}+07$ & $1.4 \mathrm{E}+02$ \\
\hline Ac-224 & $5.1 \mathrm{E}-04$ & $1.9 \mathrm{E}+12$ & $4.4 \mathrm{E}+06$ \\
\hline Ac-225 & $2.5 \mathrm{E}-02$ & $9.3 \mathrm{E}+13$ & $2.2 \mathrm{E}+08$ \\
\hline Ac-226 & 2.9E-03 & $1.1 \mathrm{E}+13$ & $2.5 \mathrm{E}+07$ \\
\hline Ac-227 & $1.0 \mathrm{E}+01$ & $3.7 \mathrm{E}+16$ & $8.7 E+10$ \\
\hline Ac-228 & $4.4 \mathrm{E}-04$ & $1.6 \mathrm{E}+12$ & $3.8 \mathrm{E}+06$ \\
\hline Ag-102 & $3.9 \mathrm{E}-06$ & $1.4 \mathrm{E}+10$ & $3.4 \mathrm{E}+04$ \\
\hline Ag-103 & $2.5 \mathrm{E}-06$ & $9.3 \mathrm{E}+09$ & $2.2 \mathrm{E}+04$ \\
\hline Ag-104 & $7.9 \mathrm{E}-06$ & $2.9 \mathrm{E}+10$ & $6.8 \mathrm{E}+04$ \\
\hline Ag-104m & $3.2 \mathrm{E}-06$ & $1.2 \mathrm{E}+10$ & $2.8 \mathrm{E}+04$ \\
\hline Ag-105 & $1.5 \mathrm{E}-03$ & $5.6 \mathrm{E}+12$ & $1.3 \mathrm{E}+07$ \\
\hline Ag-106 & $1.2 \mathrm{E}-06$ & $4.4 \mathrm{E}+09$ & $1.0 \mathrm{E}+04$ \\
\hline Ag-106m & $1.8 \mathrm{E}-03$ & $6.7 \mathrm{E}+12$ & $1.6 \mathrm{E}+07$ \\
\hline Ag-108 & $3.0 \mathrm{E}-09$ & $1.1 \mathrm{E}+07$ & $2.6 \mathrm{E}+01$ \\
\hline Ag-108m & $3.6 \mathrm{E}-01$ & $1.3 \mathrm{E}+15$ & $3.1 \mathrm{E}+09$ \\
\hline Ag-109m & $1.5 \mathrm{E}-10$ & $5.6 \mathrm{E}+05$ & $1.3 \mathrm{E}+00$ \\
\hline Ag-110 & $3.1 \mathrm{E}-10$ & $1.1 \mathrm{E}+06$ & $2.7 \mathrm{E}+00$ \\
\hline Ag-110m & $2.8 \mathrm{E}-02$ & $1.0 \mathrm{E}+14$ & $2.4 \mathrm{E}+08$ \\
\hline Ag-111 & $1.0 \mathrm{E}-03$ & $3.7 \mathrm{E}+12$ & $8.7 E+06$ \\
\hline Ag-112 & $5.1 \mathrm{E}-06$ & $1.9 \mathrm{E}+10$ & $4.4 \mathrm{E}+04$ \\
\hline Ag-115 & $2.4 \mathrm{E}-06$ & $8.9 \mathrm{E}+09$ & $2.1 \mathrm{E}+04$ \\
\hline Al-26 & $5.7 \mathrm{E}-01$ & $2.1 \mathrm{E}+15$ & $4.9 \mathrm{E}+09$ \\
\hline Al-28 & 2.7E-07 & $1.0 \mathrm{E}+09$ & $2.3 \mathrm{E}+03$ \\
\hline Am-237 & $1.4 \mathrm{E}-06$ & $5.2 \mathrm{E}+09$ & $1.2 \mathrm{E}+04$ \\
\hline Am-238 & $5.2 \mathrm{E}-06$ & $1.9 \mathrm{E}+10$ & $4.5 \mathrm{E}+04$ \\
\hline Am-239 & 7.2E-06 & $2.7 \mathrm{E}+10$ & $6.2 \mathrm{E}+04$ \\
\hline Am-240 & $1.2 \mathrm{E}-04$ & $4.4 \mathrm{E}+11$ & $1.0 \mathrm{E}+06$ \\
\hline Am-241 & $1.0 \mathrm{E}+00$ & $3.7 \mathrm{E}+15$ & $8.7 \mathrm{E}+09$ \\
\hline Am-242 & $1.4 \mathrm{E}-04$ & $5.2 \mathrm{E}+11$ & $1.2 \mathrm{E}+06$ \\
\hline Am-242m & $1.0 \mathrm{E}+00$ & $3.7 \mathrm{E}+15$ & $8.7 \mathrm{E}+09$ \\
\hline Am-243 & $1.1 \mathrm{E}+00$ & $4.1 \mathrm{E}+15$ & $9.5 \mathrm{E}+09$ \\
\hline Am-244 & $5.1 \mathrm{E}-05$ & $1.9 \mathrm{E}+11$ & $4.4 \mathrm{E}+05$ \\
\hline Am-244m & $1.5 \mathrm{E}-06$ & $5.6 \mathrm{E}+09$ & $1.3 \mathrm{E}+04$ \\
\hline Am-245 & $3.1 \mathrm{E}-07$ & $1.1 \mathrm{E}+09$ & $2.7 \mathrm{E}+03$ \\
\hline Am-246 & $2.1 \mathrm{E}-06$ & $7.8 \mathrm{E}+09$ & $1.8 \mathrm{E}+04$ \\
\hline Am-246m & $1.7 \mathrm{E}-06$ & $6.3 \mathrm{E}+09$ & $1.5 \mathrm{E}+04$ \\
\hline Ar-37 & $1.7 \mathrm{E}-12$ & $6.3 \mathrm{E}+03$ & $1.5 \mathrm{E}-02$ \\
\hline Ar-39 & $0.0 \mathrm{E}+00$ & $0.0 \mathrm{E}+00$ & $0.0 \mathrm{E}+00$ \\
\hline Ar-41 & $1.5 \mathrm{E}-06$ & $5.6 \mathrm{E}+09$ & $1.3 \mathrm{E}+04$ \\
\hline As-69 & $1.8 \mathrm{E}-06$ & $6.7 \mathrm{E}+09$ & $1.6 \mathrm{E}+04$ \\
\hline As-70 & $1.0 \mathrm{E}-05$ & $3.7 \mathrm{E}+10$ & $8.7 \mathrm{E}+04$ \\
\hline As-71 & $1.1 \mathrm{E}-04$ & $4.1 \mathrm{E}+11$ & $9.5 \mathrm{E}+05$ \\
\hline As-72 & $1.2 \mathrm{E}-04$ & $4.4 \mathrm{E}+11$ & $1.0 \mathrm{E}+06$ \\
\hline As-73 & $3.1 \mathrm{E}-04$ & $1.1 \mathrm{E}+12$ & $2.7 \mathrm{E}+06$ \\
\hline As-74 & $1.4 \mathrm{E}-03$ & $5.2 \mathrm{E}+12$ & $1.2 \mathrm{E}+07$ \\
\hline
\end{tabular}


Table 1. Results of the Screening Analysis

\begin{tabular}{|c|c|c|c|}
\hline Radionuclide & $\begin{array}{c}\text { Level I } \\
\text { Screening Factor } \\
\left(\mathrm{Sv} / \mathrm{Bq}-\mathrm{m}^{-3}\right) \\
\end{array}$ & $\begin{array}{c}\text { Level I } \\
\text { Screening Factor } \\
\left(\mathrm{mrem} / \mathrm{Ci}-\mathrm{m}^{-3}\right) \\
\end{array}$ & $\begin{array}{l}\text { Screening } \\
\text { Dose } \\
\text { mrem/yr } \\
\end{array}$ \\
\hline As-76 & $6.2 \mathrm{E}-05$ & $2.3 \mathrm{E}+11$ & $5.4 \mathrm{E}+05$ \\
\hline As-77 & $2.1 \mathrm{E}-05$ & $7.8 \mathrm{E}+10$ & $1.8 \mathrm{E}+05$ \\
\hline As-78 & $4.8 \mathrm{E}-06$ & $1.8 \mathrm{E}+10$ & $4.2 \mathrm{E}+04$ \\
\hline At-207 & $1.6 \mathrm{E}-05$ & $5.9 \mathrm{E}+10$ & $1.4 \mathrm{E}+05$ \\
\hline At-211 & $2.4 \mathrm{E}-04$ & $8.9 \mathrm{E}+11$ & $2.1 \mathrm{E}+06$ \\
\hline At-215 & $5.8 \mathrm{E}-15$ & $2.1 \mathrm{E}+01$ & $5.0 \mathrm{E}-05$ \\
\hline At-216 & $3.1 \mathrm{E}-12$ & $1.1 \mathrm{E}+04$ & 2.7E-02 \\
\hline At-217 & $3.2 \mathrm{E}-10$ & $1.2 \mathrm{E}+06$ & $2.8 \mathrm{E}+00$ \\
\hline At-218 & $2.2 \mathrm{E}-08$ & $8.1 \mathrm{E}+07$ & $1.9 \mathrm{E}+02$ \\
\hline $\mathrm{Au}-193$ & 7.0E-06 & $2.6 \mathrm{E}+10$ & $6.1 \mathrm{E}+04$ \\
\hline Au-194 & 8.2E-05 & $3.0 \mathrm{E}+11$ & $7.1 \mathrm{E}+05$ \\
\hline $\mathrm{Au}-195$ & $9.1 \mathrm{E}-04$ & $3.4 \mathrm{E}+12$ & $7.9 \mathrm{E}+06$ \\
\hline $\mathrm{Au}-195 \mathrm{~m}$ & $4.5 \mathrm{E}-09$ & $1.7 \mathrm{E}+07$ & $3.9 \mathrm{E}+01$ \\
\hline Au-198 & $1.5 \mathrm{E}-04$ & $5.6 \mathrm{E}+11$ & $1.3 \mathrm{E}+06$ \\
\hline $\mathrm{Au}-198 \mathrm{~m}$ & $2.8 \mathrm{E}-04$ & $1.0 \mathrm{E}+12$ & $2.4 \mathrm{E}+06$ \\
\hline Au-199 & $6.9 \mathrm{E}-05$ & $2.6 \mathrm{E}+11$ & $6.0 \mathrm{E}+05$ \\
\hline $\mathrm{Au}-200$ & $8.1 \mathrm{E}-07$ & $3.0 \mathrm{E}+09$ & $7.0 \mathrm{E}+03$ \\
\hline $\mathrm{Au}-200 \mathrm{~m}$ & $8.3 \mathrm{E}-05$ & $3.1 \mathrm{E}+11$ & $7.2 \mathrm{E}+05$ \\
\hline $\mathrm{Au}-201$ & $1.4 \mathrm{E}-07$ & $5.2 \mathrm{E}+08$ & $1.2 \mathrm{E}+03$ \\
\hline Ba-126 & $5.4 \mathrm{E}-06$ & $2.0 \mathrm{E}+10$ & $4.7 \mathrm{E}+04$ \\
\hline Ba-128 & $3.3 \mathrm{E}-04$ & $1.2 \mathrm{E}+12$ & $2.9 \mathrm{E}+06$ \\
\hline Ba-131 & 4.2E-04 & $1.6 \mathrm{E}+12$ & $3.6 \mathrm{E}+06$ \\
\hline Ba-131m & $4.6 \mathrm{E}-07$ & $1.7 \mathrm{E}+09$ & $4.0 \mathrm{E}+03$ \\
\hline Ba-133 & $4.6 \mathrm{E}-02$ & $1.7 \mathrm{E}+14$ & $4.0 \mathrm{E}+08$ \\
\hline Ba-133m & $5.2 \mathrm{E}-05$ & $1.9 \mathrm{E}+11$ & $4.5 \mathrm{E}+05$ \\
\hline Ba-135m & $1.8 \mathrm{E}-05$ & $6.7 \mathrm{E}+10$ & $1.6 \mathrm{E}+05$ \\
\hline Ba-137m & $1.2 \mathrm{E}-07$ & $4.4 \mathrm{E}+08$ & $1.0 \mathrm{E}+03$ \\
\hline Ba-139 & $4.6 \mathrm{E}-07$ & $1.7 \mathrm{E}+09$ & $4.0 \mathrm{E}+03$ \\
\hline Ba-140 & $2.4 \mathrm{E}-03$ & $8.9 \mathrm{E}+12$ & $2.1 \mathrm{E}+07$ \\
\hline Ba-141 & $1.6 \mathrm{E}-06$ & $5.9 \mathrm{E}+09$ & $1.4 \mathrm{E}+04$ \\
\hline Ba-142 & $1.8 \mathrm{E}-06$ & $6.7 \mathrm{E}+09$ & $1.6 \mathrm{E}+04$ \\
\hline Be-7 & $1.2 \mathrm{E}-04$ & $4.4 \mathrm{E}+11$ & $1.0 \mathrm{E}+06$ \\
\hline Be-10 & $2.6 \mathrm{E}-03$ & $9.6 \mathrm{E}+12$ & $2.2 \mathrm{E}+07$ \\
\hline $\mathrm{Bi}-200$ & $6.6 \mathrm{E}-06$ & $2.4 \mathrm{E}+10$ & $5.7 \mathrm{E}+04$ \\
\hline Bi-201 & $8.4 \mathrm{E}-06$ & $3.1 \mathrm{E}+10$ & $7.3 \mathrm{E}+04$ \\
\hline Bi-202 & $1.0 \mathrm{E}-05$ & $3.7 \mathrm{E}+10$ & $8.7 \mathrm{E}+04$ \\
\hline $\mathrm{Bi}-203$ & 5.7E-05 & $2.1 \mathrm{E}+11$ & $4.9 \mathrm{E}+05$ \\
\hline Bi-205 & $1.3 \mathrm{E}-03$ & $4.8 \mathrm{E}+12$ & $1.1 \mathrm{E}+07$ \\
\hline Bi-206 & $1.2 \mathrm{E}-03$ & $4.4 \mathrm{E}+12$ & $1.0 \mathrm{E}+07$ \\
\hline Bi-207 & $2.7 \mathrm{E}-01$ & $1.0 \mathrm{E}+15$ & $2.3 \mathrm{E}+09$ \\
\hline $\mathrm{Bi}-210$ & $4.5 \mathrm{E}-03$ & $1.7 \mathrm{E}+13$ & $3.9 \mathrm{E}+07$ \\
\hline $\mathrm{Bi}-210 \mathrm{~m}$ & $1.2 \mathrm{E}-01$ & $4.4 \mathrm{E}+14$ & $1.0 \mathrm{E}+09$ \\
\hline Bi-211 & 7.4E-09 & $2.7 \mathrm{E}+07$ & $6.4 \mathrm{E}+01$ \\
\hline $\mathrm{Bi}-212$ & $3.8 \mathrm{E}-05$ & $1.4 \mathrm{E}+11$ & $3.3 \mathrm{E}+05$ \\
\hline $\mathrm{Bi}-213$ & 2.7E-05 & $1.0 \mathrm{E}+11$ & $2.3 \mathrm{E}+05$ \\
\hline Bi-214 & $1.3 \mathrm{E}-05$ & $4.8 \mathrm{E}+10$ & $1.1 \mathrm{E}+05$ \\
\hline
\end{tabular}


Table 1. Results of the Screening Analysis

\begin{tabular}{|c|c|c|c|}
\hline Radionuclide & $\begin{array}{c}\text { Level I } \\
\text { Screening Factor } \\
\left(\mathrm{Sv} / \mathrm{Bq}-\mathrm{m}^{-3}\right) \\
\end{array}$ & $\begin{array}{c}\text { Level I } \\
\text { Screening Factor } \\
\left(\mathrm{mrem} / \mathrm{Ci}-\mathrm{m}^{-3}\right) \\
\end{array}$ & $\begin{array}{l}\text { Screening } \\
\text { Dose } \\
\text { mrem/yr } \\
\end{array}$ \\
\hline Bk-245 & $1.5 \mathrm{E}-04$ & $5.6 \mathrm{E}+11$ & $1.3 \mathrm{E}+06$ \\
\hline Bk-246 & $9.0 \mathrm{E}-05$ & $3.3 \mathrm{E}+11$ & $7.8 \mathrm{E}+05$ \\
\hline Bk-247 & $1.3 \mathrm{E}+00$ & $4.8 \mathrm{E}+15$ & $1.1 \mathrm{E}+10$ \\
\hline Bk-249 & $3.3 \mathrm{E}-03$ & $1.2 \mathrm{E}+13$ & $2.9 \mathrm{E}+07$ \\
\hline Bk-250 & $2.0 \mathrm{E}-05$ & $7.4 \mathrm{E}+10$ & $1.7 \mathrm{E}+05$ \\
\hline Br-74 & 7.9E-06 & $2.9 \mathrm{E}+10$ & $6.8 \mathrm{E}+04$ \\
\hline Br-74m & $9.2 \mathrm{E}-06$ & $3.4 \mathrm{E}+10$ & $8.0 \mathrm{E}+04$ \\
\hline $\mathrm{Br}-75$ & $1.2 \mathrm{E}-05$ & $4.4 \mathrm{E}+10$ & $1.0 \mathrm{E}+05$ \\
\hline $\mathrm{Br}-76$ & $8.1 \mathrm{E}-05$ & $3.0 \mathrm{E}+11$ & $7.0 \mathrm{E}+05$ \\
\hline Br-77 & $6.4 \mathrm{E}-05$ & $2.4 \mathrm{E}+11$ & $5.5 \mathrm{E}+05$ \\
\hline $\mathrm{Br}-80$ & $1.5 \mathrm{E}-07$ & $5.6 \mathrm{E}+08$ & $1.3 \mathrm{E}+03$ \\
\hline $\mathrm{Br}-80 \mathrm{~m}$ & $1.6 \mathrm{E}-06$ & $5.9 \mathrm{E}+09$ & $1.4 \mathrm{E}+04$ \\
\hline Br-82 & 2.3E-04 & $8.5 E+11$ & $2.0 \mathrm{E}+06$ \\
\hline $\mathrm{Br}-83$ & $2.4 \mathrm{E}-07$ & $8.9 \mathrm{E}+08$ & $2.1 \mathrm{E}+03$ \\
\hline $\mathrm{Br}-84$ & $3.6 \mathrm{E}-06$ & $1.3 \mathrm{E}+10$ & $3.1 \mathrm{E}+04$ \\
\hline C-11 & $1.5 \mathrm{E}-06$ & $5.6 \mathrm{E}+09$ & $1.3 \mathrm{E}+04$ \\
\hline C-14 & $2.6 \mathrm{E}-04$ & $9.6 \mathrm{E}+11$ & $2.2 \mathrm{E}+06$ \\
\hline $\mathrm{Ca}-41$ & $2.4 \mathrm{E}-03$ & $8.9 \mathrm{E}+12$ & $2.1 \mathrm{E}+07$ \\
\hline $\mathrm{Ca}-45$ & $1.0 \mathrm{E}-03$ & $3.7 \mathrm{E}+12$ & $8.7 E+06$ \\
\hline $\mathrm{Ca}-47$ & 7.3E-04 & $2.7 \mathrm{E}+12$ & $6.3 \mathrm{E}+06$ \\
\hline $\mathrm{Ca}-49$ & $3.0 \mathrm{E}-06$ & $1.1 \mathrm{E}+10$ & $2.6 \mathrm{E}+04$ \\
\hline Cd-104 & 4.9E-06 & $1.8 \mathrm{E}+10$ & $4.2 \mathrm{E}+04$ \\
\hline Cd-107 & $6.1 \mathrm{E}-07$ & $2.3 \mathrm{E}+09$ & $5.3 \mathrm{E}+03$ \\
\hline Cd-109 & $2.8 \mathrm{E}-03$ & $1.0 \mathrm{E}+13$ & $2.4 \mathrm{E}+07$ \\
\hline Cd-113 & $1.5 \mathrm{E}-01$ & $5.6 \mathrm{E}+14$ & $1.3 \mathrm{E}+09$ \\
\hline Cd-113m & 8.3E-02 & $3.1 \mathrm{E}+14$ & 7.2E+08 \\
\hline Cd-115 & $2.0 \mathrm{E}-04$ & $7.4 \mathrm{E}+11$ & $1.7 E+06$ \\
\hline $\mathrm{Cd}-115 \mathrm{~m}$ & $2.8 \mathrm{E}-03$ & $1.0 \mathrm{E}+13$ & $2.4 \mathrm{E}+07$ \\
\hline Cd-117 & $4.1 \mathrm{E}-05$ & $1.5 \mathrm{E}+11$ & $3.5 \mathrm{E}+05$ \\
\hline $\mathrm{Cd}-117 \mathrm{~m}$ & $1.7 \mathrm{E}-05$ & $6.3 \mathrm{E}+10$ & $1.5 \mathrm{E}+05$ \\
\hline $\mathrm{Ce}-134$ & $3.6 \mathrm{E}-04$ & $1.3 \mathrm{E}+12$ & $3.1 \mathrm{E}+06$ \\
\hline Ce-135 & $6.5 \mathrm{E}-05$ & $2.4 \mathrm{E}+11$ & $5.6 \mathrm{E}+05$ \\
\hline Ce-137 & $6.6 \mathrm{E}-07$ & $2.4 \mathrm{E}+09$ & $5.7 E+03$ \\
\hline $\mathrm{Ce}-137 \mathrm{~m}$ & $3.0 \mathrm{E}-05$ & $1.1 \mathrm{E}+11$ & $2.6 \mathrm{E}+05$ \\
\hline Ce-139 & $1.0 \mathrm{E}-03$ & $3.7 \mathrm{E}+12$ & $8.7 E+06$ \\
\hline Ce-141 & $5.0 \mathrm{E}-04$ & $1.9 \mathrm{E}+12$ & $4.3 \mathrm{E}+06$ \\
\hline $\mathrm{Ce}-143$ & $1.1 \mathrm{E}-04$ & $4.1 \mathrm{E}+11$ & $9.5 \mathrm{E}+05$ \\
\hline Ce-144 & $5.2 \mathrm{E}-03$ & $1.9 \mathrm{E}+13$ & $4.5 \mathrm{E}+07$ \\
\hline Cf-244 & $2.0 \mathrm{E}-05$ & $7.4 \mathrm{E}+10$ & $1.7 \mathrm{E}+05$ \\
\hline Cf-246 & $1.5 \mathrm{E}-03$ & $5.6 \mathrm{E}+12$ & $1.3 \mathrm{E}+07$ \\
\hline Cf-248 & $1.3 \mathrm{E}-01$ & $4.8 \mathrm{E}+14$ & $1.1 \mathrm{E}+09$ \\
\hline Cf-249 & $1.3 \mathrm{E}+00$ & $4.8 \mathrm{E}+15$ & $1.1 \mathrm{E}+10$ \\
\hline Cf-250 & 5.7E-01 & $2.1 \mathrm{E}+15$ & $4.9 \mathrm{E}+09$ \\
\hline Cf- 251 & $1.3 \mathrm{E}+00$ & $4.8 \mathrm{E}+15$ & $1.1 \mathrm{E}+10$ \\
\hline Cf-252 & $4.1 \mathrm{E}-01$ & $1.5 \mathrm{E}+15$ & $3.5 \mathrm{E}+09$ \\
\hline Cf-253 & $8.5 \mathrm{E}-03$ & $3.1 \mathrm{E}+13$ & $7.4 \mathrm{E}+07$ \\
\hline
\end{tabular}


Table 1. Results of the Screening Analysis

\begin{tabular}{|c|c|c|c|}
\hline Radionuclide & $\begin{array}{c}\text { Level I } \\
\text { Screening Factor } \\
\left(\mathrm{Sv} / \mathrm{Bq}-\mathrm{m}^{-3}\right) \\
\end{array}$ & $\begin{array}{c}\text { Level I } \\
\text { Screening Factor } \\
\left(\mathrm{mrem} / \mathrm{Ci}-\mathrm{m}^{-3}\right) \\
\end{array}$ & $\begin{array}{l}\text { Screening } \\
\text { Dose } \\
\text { mrem/yr } \\
\end{array}$ \\
\hline Cf-254 & 8.7E-01 & $3.2 \mathrm{E}+15$ & $7.5 \mathrm{E}+09$ \\
\hline $\mathrm{Cl}-36$ & $6.5 \mathrm{E}-01$ & $2.4 \mathrm{E}+15$ & $5.6 \mathrm{E}+09$ \\
\hline $\mathrm{Cl}-38$ & 3.3E-06 & $1.2 \mathrm{E}+10$ & $2.9 \mathrm{E}+04$ \\
\hline $\mathrm{Cl}-39$ & $3.8 \mathrm{E}-06$ & $1.4 \mathrm{E}+10$ & $3.3 \mathrm{E}+04$ \\
\hline $\mathrm{Cm}-238$ & $1.6 \mathrm{E}-05$ & $5.9 \mathrm{E}+10$ & $1.4 \mathrm{E}+05$ \\
\hline $\mathrm{Cm}-240$ & $1.9 \mathrm{E}-02$ & $7.0 \mathrm{E}+13$ & $1.6 \mathrm{E}+08$ \\
\hline $\mathrm{Cm}-241$ & $1.4 \mathrm{E}-03$ & $5.2 \mathrm{E}+12$ & $1.2 \mathrm{E}+07$ \\
\hline $\mathrm{Cm}-242$ & 4.0E-02 & $1.5 \mathrm{E}+14$ & $3.5 \mathrm{E}+08$ \\
\hline $\mathrm{Cm}-243$ & $7.1 \mathrm{E}-01$ & $2.6 \mathrm{E}+15$ & $6.1 \mathrm{E}+09$ \\
\hline $\mathrm{Cm}-244$ & $5.4 \mathrm{E}-01$ & $2.0 \mathrm{E}+15$ & 4.7E+09 \\
\hline $\mathrm{Cm}-245$ & $1.1 \mathrm{E}+00$ & $4.1 \mathrm{E}+15$ & $9.5 \mathrm{E}+09$ \\
\hline $\mathrm{Cm}-246$ & $1.1 \mathrm{E}+00$ & $4.1 \mathrm{E}+15$ & $9.5 \mathrm{E}+09$ \\
\hline $\mathrm{Cm}-247$ & $1.1 \mathrm{E}+00$ & $4.1 \mathrm{E}+15$ & $9.5 \mathrm{E}+09$ \\
\hline $\mathrm{Cm}-248$ & $3.9 \mathrm{E}+00$ & $1.4 \mathrm{E}+16$ & $3.4 \mathrm{E}+10$ \\
\hline $\mathrm{Cm}-249$ & $5.5 \mathrm{E}-07$ & $2.0 \mathrm{E}+09$ & $4.8 \mathrm{E}+03$ \\
\hline $\mathrm{Cm}-250$ & $2.2 \mathrm{E}+01$ & $8.1 \mathrm{E}+16$ & $1.9 \mathrm{E}+11$ \\
\hline Co-55 & $7.5 \mathrm{E}-05$ & $2.8 \mathrm{E}+11$ & $6.5 \mathrm{E}+05$ \\
\hline Co-56 & $1.6 \mathrm{E}-02$ & $5.9 \mathrm{E}+13$ & $1.4 \mathrm{E}+08$ \\
\hline Co-57 & $2.1 \mathrm{E}-03$ & $7.8 \mathrm{E}+12$ & $1.8 \mathrm{E}+07$ \\
\hline Co-58 & $4.2 \mathrm{E}-03$ & $1.6 \mathrm{E}+13$ & $3.6 \mathrm{E}+07$ \\
\hline Co-58m & $2.2 \mathrm{E}-05$ & $8.1 \mathrm{E}+10$ & $1.9 \mathrm{E}+05$ \\
\hline Co-60 & $1.7 \mathrm{E}-01$ & $6.3 \mathrm{E}+14$ & $1.5 \mathrm{E}+09$ \\
\hline Co-60m & $6.4 \mathrm{E}-07$ & $2.4 \mathrm{E}+09$ & $5.5 \mathrm{E}+03$ \\
\hline Co-61 & $5.6 \mathrm{E}-07$ & $2.1 \mathrm{E}+09$ & $4.8 \mathrm{E}+03$ \\
\hline Co- $62 \mathrm{~m}$ & 3.3E-06 & $1.2 \mathrm{E}+10$ & $2.9 \mathrm{E}+04$ \\
\hline $\mathrm{Cr}-48$ & $1.8 \mathrm{E}-04$ & $6.7 \mathrm{E}+11$ & $1.6 \mathrm{E}+06$ \\
\hline Cr-49 & 2.3E-06 & $8.5 \mathrm{E}+09$ & $2.0 \mathrm{E}+04$ \\
\hline $\mathrm{Cr}-51$ & $9.2 \mathrm{E}-05$ & $3.4 \mathrm{E}+11$ & $8.0 \mathrm{E}+05$ \\
\hline Cs-125 & 2.7E-05 & $1.0 \mathrm{E}+11$ & $2.3 \mathrm{E}+05$ \\
\hline Cs-126 & $8.8 \mathrm{E}-08$ & $3.3 \mathrm{E}+08$ & $7.6 \mathrm{E}+02$ \\
\hline Cs-127 & 7.5E-06 & $2.8 \mathrm{E}+10$ & $6.5 \mathrm{E}+04$ \\
\hline Cs-128 & $3.5 \mathrm{E}-07$ & $1.3 \mathrm{E}+09$ & $3.0 \mathrm{E}+03$ \\
\hline Cs-129 & $2.1 \mathrm{E}-05$ & $7.8 \mathrm{E}+10$ & $1.8 \mathrm{E}+05$ \\
\hline Cs-130 & 9.8E-07 & $3.6 \mathrm{E}+09$ & $8.5 \mathrm{E}+03$ \\
\hline Cs-131 & $1.1 \mathrm{E}-04$ & $4.1 \mathrm{E}+11$ & $9.5 \mathrm{E}+05$ \\
\hline Cs-132 & 7.0E-04 & $2.6 \mathrm{E}+12$ & $6.1 \mathrm{E}+06$ \\
\hline Cs-134 & $1.3 \mathrm{E}-01$ & $4.8 \mathrm{E}+14$ & $1.1 \mathrm{E}+09$ \\
\hline Cs-134m & $2.1 \mathrm{E}-05$ & $7.8 \mathrm{E}+10$ & $1.8 \mathrm{E}+05$ \\
\hline Cs-135 & $2.0 \mathrm{E}-02$ & $7.4 \mathrm{E}+13$ & $1.7 \mathrm{E}+08$ \\
\hline Cs-135m & $4.0 \mathrm{E}-06$ & $1.5 \mathrm{E}+10$ & $3.5 \mathrm{E}+04$ \\
\hline Cs-136 & $6.7 \mathrm{E}-03$ & $2.5 E+13$ & $5.8 \mathrm{E}+07$ \\
\hline Cs-137 & $2.2 \mathrm{E}-01$ & $8.1 \mathrm{E}+14$ & $1.9 \mathrm{E}+09$ \\
\hline Cs-138 & $4.5 \mathrm{E}-06$ & $1.7 \mathrm{E}+10$ & $3.9 \mathrm{E}+04$ \\
\hline $\mathrm{Cu}-60$ & $6.3 \mathrm{E}-06$ & $2.3 \mathrm{E}+10$ & $5.5 \mathrm{E}+04$ \\
\hline $\mathrm{Cu}-61$ & 5.7E-06 & $2.1 \mathrm{E}+10$ & $4.9 \mathrm{E}+04$ \\
\hline $\mathrm{Cu}-62$ & $9.3 \mathrm{E}-07$ & $3.4 \mathrm{E}+09$ & $8.0 \mathrm{E}+03$ \\
\hline
\end{tabular}


Table 1. Results of the Screening Analysis

\begin{tabular}{|c|c|c|c|}
\hline Radionuclide & $\begin{array}{c}\text { Level I } \\
\text { Screening Factor } \\
\left(\mathrm{Sv} / \mathrm{Bq}-\mathrm{m}^{-3}\right) \\
\end{array}$ & $\begin{array}{c}\text { Level I } \\
\text { Screening Factor } \\
\left(\mathrm{mrem} / \mathrm{Ci}-\mathrm{m}^{-3}\right) \\
\end{array}$ & $\begin{array}{l}\text { Screening } \\
\text { Dose } \\
\text { mrem/yr } \\
\end{array}$ \\
\hline $\mathrm{Cu}-64$ & 5.2E-06 & $1.9 \mathrm{E}+10$ & $4.5 \mathrm{E}+04$ \\
\hline $\mathrm{Cu}-66$ & $4.6 \mathrm{E}-08$ & $1.7 \mathrm{E}+08$ & $4.0 \mathrm{E}+02$ \\
\hline $\mathrm{Cu}-67$ & $6.3 \mathrm{E}-05$ & $2.3 \mathrm{E}+11$ & $5.5 \mathrm{E}+05$ \\
\hline Dy-155 & $1.6 \mathrm{E}-05$ & $5.9 \mathrm{E}+10$ & $1.4 \mathrm{E}+05$ \\
\hline Dy-157 & $5.2 \mathrm{E}-06$ & $1.9 \mathrm{E}+10$ & $4.5 \mathrm{E}+04$ \\
\hline Dy-159 & $3.2 \mathrm{E}-04$ & $1.2 \mathrm{E}+12$ & $2.8 \mathrm{E}+06$ \\
\hline Dy-165 & 3.9E-07 & $1.4 \mathrm{E}+09$ & $3.4 \mathrm{E}+03$ \\
\hline Dy-166 & $3.6 \mathrm{E}-04$ & $1.3 \mathrm{E}+12$ & $3.1 \mathrm{E}+06$ \\
\hline Er-161 & $6.1 \mathrm{E}-06$ & $2.3 E+10$ & $5.3 \mathrm{E}+04$ \\
\hline Er-165 & $8.4 \mathrm{E}-07$ & $3.1 \mathrm{E}+09$ & $7.3 \mathrm{E}+03$ \\
\hline Er-169 & $1.3 \mathrm{E}-04$ & $4.8 \mathrm{E}+11$ & $1.1 \mathrm{E}+06$ \\
\hline Er-171 & $6.7 \mathrm{E}-06$ & $2.5 \mathrm{E}+10$ & $5.8 \mathrm{E}+04$ \\
\hline Er-172 & $2.6 \mathrm{E}-04$ & $9.6 \mathrm{E}+11$ & $2.2 \mathrm{E}+06$ \\
\hline Es-250 & $1.1 \mathrm{E}-05$ & $4.1 \mathrm{E}+10$ & $9.5 \mathrm{E}+04$ \\
\hline Es-251 & $2.2 \mathrm{E}-05$ & $8.1 \mathrm{E}+10$ & $1.9 \mathrm{E}+05$ \\
\hline Es-253 & $1.0 \mathrm{E}-02$ & $3.7 \mathrm{E}+13$ & $8.7 \mathrm{E}+07$ \\
\hline Es-254 & 9.4E-02 & $3.5 \mathrm{E}+14$ & $8.1 \mathrm{E}+08$ \\
\hline Es-254m & $1.4 \mathrm{E}-03$ & $5.2 \mathrm{E}+12$ & $1.2 \mathrm{E}+07$ \\
\hline Eu-145 & $4.6 \mathrm{E}-04$ & $1.7 \mathrm{E}+12$ & $4.0 \mathrm{E}+06$ \\
\hline Eu-146 & $6.0 \mathrm{E}-04$ & $2.2 \mathrm{E}+12$ & $5.2 \mathrm{E}+06$ \\
\hline Eu-147 & $6.6 \mathrm{E}-04$ & $2.4 \mathrm{E}+12$ & $5.7 \mathrm{E}+06$ \\
\hline Eu-148 & $3.4 \mathrm{E}-02$ & $1.3 \mathrm{E}+14$ & $2.9 \mathrm{E}+08$ \\
\hline Eu-149 & $3.0 \mathrm{E}-04$ & $1.1 \mathrm{E}+12$ & $2.6 \mathrm{E}+06$ \\
\hline Eu-150a & 4.4E-06 & $1.6 \mathrm{E}+10$ & $3.8 \mathrm{E}+04$ \\
\hline $\mathrm{Eu}-150 \mathrm{~b}$ & $2.8 \mathrm{E}-01$ & $1.0 \mathrm{E}+15$ & $2.4 \mathrm{E}+09$ \\
\hline Eu-152 & $1.4 \mathrm{E}-01$ & $5.2 \mathrm{E}+14$ & $1.2 \mathrm{E}+09$ \\
\hline $\mathrm{Eu}-152 \mathrm{~m}$ & 7.6E-06 & $2.8 \mathrm{E}+10$ & $6.6 \mathrm{E}+04$ \\
\hline Eu-154 & $1.2 \mathrm{E}-01$ & $4.4 \mathrm{E}+14$ & $1.0 \mathrm{E}+09$ \\
\hline Eu-155 & 4.7E-03 & $1.7 \mathrm{E}+13$ & $4.1 \mathrm{E}+07$ \\
\hline Eu-156 & $1.6 \mathrm{E}-03$ & $5.9 \mathrm{E}+12$ & $1.4 \mathrm{E}+07$ \\
\hline Eu-157 & $1.4 \mathrm{E}-05$ & $5.2 \mathrm{E}+10$ & $1.2 \mathrm{E}+05$ \\
\hline Eu-158 & $2.6 \mathrm{E}-06$ & $9.6 \mathrm{E}+09$ & $2.2 \mathrm{E}+04$ \\
\hline F-18 & 4.1E-06 & $1.5 \mathrm{E}+10$ & $3.5 \mathrm{E}+04$ \\
\hline $\mathrm{Fe}-52$ & 4.8E-05 & $1.8 \mathrm{E}+11$ & $4.2 \mathrm{E}+05$ \\
\hline $\mathrm{Fe}-55$ & 2.8E-04 & $1.0 \mathrm{E}+12$ & $2.4 \mathrm{E}+06$ \\
\hline $\mathrm{Fe}-59$ & 4.3E-03 & $1.6 \mathrm{E}+13$ & $3.7 \mathrm{E}+07$ \\
\hline $\mathrm{Fe}-60$ & $5.0 \mathrm{E}-01$ & $1.9 \mathrm{E}+15$ & $4.3 \mathrm{E}+09$ \\
\hline Fm-252 & $9.1 \mathrm{E}-04$ & $3.4 \mathrm{E}+12$ & $7.9 \mathrm{E}+06$ \\
\hline Fm-253 & $1.5 \mathrm{E}-03$ & $5.6 \mathrm{E}+12$ & $1.3 \mathrm{E}+07$ \\
\hline Fm-254 & 1.2E-04 & $4.4 \mathrm{E}+11$ & $1.0 \mathrm{E}+06$ \\
\hline Fm-255 & $5.9 \mathrm{E}-04$ & $2.2 \mathrm{E}+12$ & $5.1 \mathrm{E}+06$ \\
\hline Fm-257 & $5.1 \mathrm{E}-02$ & $1.9 \mathrm{E}+14$ & $4.4 \mathrm{E}+08$ \\
\hline Fr-219 & $1.2 \mathrm{E}-12$ & $4.4 \mathrm{E}+03$ & $1.0 \mathrm{E}-02$ \\
\hline Fr-220 & $2.9 \mathrm{E}-07$ & $1.1 \mathrm{E}+09$ & $2.5 \mathrm{E}+03$ \\
\hline Fr-221 & $2.1 \mathrm{E}-06$ & $7.8 \mathrm{E}+09$ & $1.8 \mathrm{E}+04$ \\
\hline Fr-222 & $1.9 \mathrm{E}-05$ & $7.0 \mathrm{E}+10$ & $1.6 \mathrm{E}+05$ \\
\hline
\end{tabular}


Table 1. Results of the Screening Analysis

\begin{tabular}{|c|c|c|c|}
\hline Radionuclide & $\begin{array}{c}\text { Level I } \\
\text { Screening Factor } \\
\left(\mathrm{Sv} / \mathrm{Bq}-\mathrm{m}^{-3}\right)\end{array}$ & $\begin{array}{c}\text { Level I } \\
\text { Screening Factor } \\
\left(\mathrm{mrem} / \mathrm{Ci}-\mathrm{m}^{-3}\right)\end{array}$ & $\begin{array}{l}\text { Screening } \\
\text { Dose } \\
\text { mrem/yr }\end{array}$ \\
\hline Fr-223 & $6.8 \mathrm{E}-05$ & $2.5 \mathrm{E}+11$ & $5.9 \mathrm{E}+05$ \\
\hline Ga-65 & $2.7 \mathrm{E}-06$ & $1.0 \mathrm{E}+10$ & $2.3 \mathrm{E}+04$ \\
\hline Ga-66 & $4.2 \mathrm{E}-05$ & $1.6 \mathrm{E}+11$ & $3.6 \mathrm{E}+05$ \\
\hline Ga-67 & $3.9 \mathrm{E}-05$ & $1.4 \mathrm{E}+11$ & $3.4 \mathrm{E}+05$ \\
\hline Ga-68 & $3.0 \mathrm{E}-06$ & $1.1 \mathrm{E}+10$ & $2.6 \mathrm{E}+04$ \\
\hline Ga-70 & $6.9 \mathrm{E}-08$ & $2.6 \mathrm{E}+08$ & $6.0 \mathrm{E}+02$ \\
\hline Ga-72 & $6.9 \mathrm{E}-05$ & $2.6 \mathrm{E}+11$ & $6.0 \mathrm{E}+05$ \\
\hline Ga-73 & $3.7 E-06$ & $1.4 \mathrm{E}+10$ & $3.2 \mathrm{E}+04$ \\
\hline Gd-145 & 4.9E-06 & $1.8 \mathrm{E}+10$ & $4.2 \mathrm{E}+04$ \\
\hline Gd-146 & 5.9E-03 & $2.2 \mathrm{E}+13$ & $5.1 \mathrm{E}+07$ \\
\hline Gd-147 & $1.5 \mathrm{E}-04$ & $5.6 \mathrm{E}+11$ & $1.3 \mathrm{E}+06$ \\
\hline Gd-148 & $4.4 \mathrm{E}-01$ & $1.6 \mathrm{E}+15$ & $3.8 \mathrm{E}+09$ \\
\hline Gd-149 & $3.2 \mathrm{E}-04$ & $1.2 \mathrm{E}+12$ & $2.8 \mathrm{E}+06$ \\
\hline Gd-151 & $4.5 \mathrm{E}-04$ & $1.7 \mathrm{E}+12$ & $3.9 \mathrm{E}+06$ \\
\hline Gd-152 & $3.2 \mathrm{E}-01$ & $1.2 \mathrm{E}+15$ & $2.8 \mathrm{E}+09$ \\
\hline Gd-153 & $1.3 \mathrm{E}-03$ & $4.8 \mathrm{E}+12$ & $1.1 \mathrm{E}+07$ \\
\hline Gd-159 & $1.0 \mathrm{E}-05$ & $3.7 \mathrm{E}+10$ & $8.7 \mathrm{E}+04$ \\
\hline Ge-66 & $1.2 \mathrm{E}-05$ & $4.4 \mathrm{E}+10$ & $1.0 \mathrm{E}+05$ \\
\hline Ge-67 & $2.2 \mathrm{E}-06$ & $8.1 \mathrm{E}+09$ & $1.9 \mathrm{E}+04$ \\
\hline Ge-68 & $1.3 \mathrm{E}-02$ & $4.8 \mathrm{E}+13$ & $1.1 \mathrm{E}+08$ \\
\hline Ge-69 & $7.2 \mathrm{E}-05$ & $2.7 \mathrm{E}+11$ & $6.2 \mathrm{E}+05$ \\
\hline Ge-71 & $1.1 \mathrm{E}-05$ & $4.1 \mathrm{E}+10$ & $9.5 \mathrm{E}+04$ \\
\hline $\mathrm{Ge}-75$ & $2.7 \mathrm{E}-07$ & $1.0 \mathrm{E}+09$ & $2.3 \mathrm{E}+03$ \\
\hline $\mathrm{Ge}-77$ & $2.8 \mathrm{E}-05$ & $1.0 \mathrm{E}+11$ & $2.4 \mathrm{E}+05$ \\
\hline Ge-78 & 4.3E-06 & $1.6 \mathrm{E}+10$ & $3.7 \mathrm{E}+04$ \\
\hline $\mathrm{H}-3$ & $1.9 \mathrm{E}-06$ & $7.0 \mathrm{E}+09$ & $1.6 \mathrm{E}+04$ \\
\hline Hf-170 & $9.4 \mathrm{E}-05$ & $3.5 \mathrm{E}+11$ & $8.1 \mathrm{E}+05$ \\
\hline Hf-172 & 4.9E-02 & $1.8 \mathrm{E}+14$ & $4.2 \mathrm{E}+08$ \\
\hline Hf-173 & $2.8 \mathrm{E}-05$ & $1.0 \mathrm{E}+11$ & $2.4 \mathrm{E}+05$ \\
\hline Hf-174 & $0.0 \mathrm{E}+00$ & $0.0 \mathrm{E}+00$ & $0.0 \mathrm{E}+00$ \\
\hline Hf- 175 & $1.2 \mathrm{E}-03$ & $4.4 \mathrm{E}+12$ & $1.0 \mathrm{E}+07$ \\
\hline Hf- $177 \mathrm{~m}$ & $5.8 \mathrm{E}-06$ & $2.1 \mathrm{E}+10$ & $5.0 \mathrm{E}+04$ \\
\hline Hf-178m & $4.5 \mathrm{E}-01$ & $1.7 \mathrm{E}+15$ & $3.9 \mathrm{E}+09$ \\
\hline Hf-179m & $1.5 \mathrm{E}-03$ & $5.6 \mathrm{E}+12$ & $1.3 \mathrm{E}+07$ \\
\hline Hf-180m & $1.1 \mathrm{E}-05$ & $4.1 \mathrm{E}+10$ & $9.5 \mathrm{E}+04$ \\
\hline Hf-181 & $1.5 \mathrm{E}-03$ & $5.6 \mathrm{E}+12$ & $1.3 \mathrm{E}+07$ \\
\hline Hf-182 & $3.5 \mathrm{E}-01$ & $1.3 \mathrm{E}+15$ & $3.0 \mathrm{E}+09$ \\
\hline Hf- $182 \mathrm{~m}$ & $3.9 \mathrm{E}-06$ & $1.4 \mathrm{E}+10$ & $3.4 \mathrm{E}+04$ \\
\hline Hf-183 & 4.8E-06 & $1.8 \mathrm{E}+10$ & $4.2 \mathrm{E}+04$ \\
\hline Hf-184 & $1.5 \mathrm{E}-05$ & $5.6 \mathrm{E}+10$ & $1.3 \mathrm{E}+05$ \\
\hline $\mathrm{Hg}-193$ & $3.1 \mathrm{E}-06$ & $1.1 \mathrm{E}+10$ & $2.7 \mathrm{E}+04$ \\
\hline $\mathrm{Hg}-193 \mathrm{~m}$ & 2.7E-05 & $1.0 \mathrm{E}+11$ & $2.3 \mathrm{E}+05$ \\
\hline Hg-194 & 4.7E-01 & $1.7 \mathrm{E}+15$ & $4.1 \mathrm{E}+09$ \\
\hline $\mathrm{Hg}-195$ & $6.1 \mathrm{E}-06$ & $2.3 \mathrm{E}+10$ & $5.3 \mathrm{E}+04$ \\
\hline $\mathrm{Hg}-195 \mathrm{~m}$ & 6.9E-05 & $2.6 \mathrm{E}+11$ & $6.0 \mathrm{E}+05$ \\
\hline Hg-197 & $3.7 \mathrm{E}-05$ & $1.4 \mathrm{E}+11$ & $3.2 \mathrm{E}+05$ \\
\hline
\end{tabular}


Table 1. Results of the Screening Analysis

\begin{tabular}{|c|c|c|c|}
\hline Radionuclide & $\begin{array}{c}\text { Level I } \\
\text { Screening Factor } \\
\left(\mathrm{Sv} / \mathrm{Bq}-\mathrm{m}^{-3}\right) \\
\end{array}$ & $\begin{array}{c}\text { Level I } \\
\text { Screening Factor } \\
\left(\mathrm{mrem} / \mathrm{Ci}-\mathrm{m}^{-3}\right) \\
\end{array}$ & $\begin{array}{l}\text { Screening } \\
\text { Dose } \\
\text { mrem/yr } \\
\end{array}$ \\
\hline Hg-197m & $3.0 \mathrm{E}-05$ & $1.1 \mathrm{E}+11$ & $2.6 \mathrm{E}+05$ \\
\hline $\mathrm{Hg}-199 \mathrm{~m}$ & $5.5 \mathrm{E}-07$ & $2.0 \mathrm{E}+09$ & $4.8 \mathrm{E}+03$ \\
\hline $\mathrm{Hg}-203$ & $1.8 \mathrm{E}-03$ & $6.7 \mathrm{E}+12$ & $1.6 \mathrm{E}+07$ \\
\hline Но- 155 & $2.2 \mathrm{E}-06$ & $8.1 \mathrm{E}+09$ & $1.9 \mathrm{E}+04$ \\
\hline Нo- 157 & $6.8 \mathrm{E}-07$ & $2.5 \mathrm{E}+09$ & $5.9 \mathrm{E}+03$ \\
\hline Но-159 & 7.7E-07 & $2.9 \mathrm{E}+09$ & $6.7 E+03$ \\
\hline Но-161 & $3.4 \mathrm{E}-07$ & $1.3 \mathrm{E}+09$ & $2.9 \mathrm{E}+03$ \\
\hline Нo-162 & $2.0 \mathrm{E}-07$ & $7.4 \mathrm{E}+08$ & $1.7 \mathrm{E}+03$ \\
\hline Но- $162 \mathrm{~m}$ & $1.9 \mathrm{E}-06$ & $7.0 \mathrm{E}+09$ & $1.6 \mathrm{E}+04$ \\
\hline Но-164 & $6.4 \mathrm{E}-08$ & $2.4 \mathrm{E}+08$ & $5.5 \mathrm{E}+02$ \\
\hline Но- $164 \mathrm{~m}$ & $1.6 \mathrm{E}-07$ & $5.9 \mathrm{E}+08$ & $1.4 \mathrm{E}+03$ \\
\hline Но-166 & $4.7 E-05$ & $1.7 \mathrm{E}+11$ & $4.1 \mathrm{E}+05$ \\
\hline Ho-166m & $3.8 \mathrm{E}-01$ & $1.4 \mathrm{E}+15$ & $3.3 \mathrm{E}+09$ \\
\hline Но-167 & $2.5 \mathrm{E}-06$ & $9.3 \mathrm{E}+09$ & $2.2 \mathrm{E}+04$ \\
\hline $\mathrm{I}-120$ & $9.9 \mathrm{E}-06$ & $3.7 \mathrm{E}+10$ & $8.6 \mathrm{E}+04$ \\
\hline $\mathrm{I}-120 \mathrm{~m}$ & $1.4 \mathrm{E}-05$ & $5.2 \mathrm{E}+10$ & $1.2 \mathrm{E}+05$ \\
\hline $\mathrm{I}-121$ & $5.1 \mathrm{E}-06$ & $1.9 \mathrm{E}+10$ & $4.4 \mathrm{E}+04$ \\
\hline $\mathrm{I}-122$ & $3.3 \mathrm{E}-07$ & $1.2 \mathrm{E}+09$ & $2.9 \mathrm{E}+03$ \\
\hline $\mathrm{I}-123$ & $7.6 \mathrm{E}-06$ & $2.8 \mathrm{E}+10$ & $6.6 \mathrm{E}+04$ \\
\hline $\mathrm{I}-124$ & $8.4 \mathrm{E}-03$ & $3.1 \mathrm{E}+13$ & $7.3 \mathrm{E}+07$ \\
\hline $\mathrm{I}-125$ & $5.2 \mathrm{E}-02$ & $1.9 \mathrm{E}+14$ & $4.5 \mathrm{E}+08$ \\
\hline $\mathrm{I}-126$ & $5.5 \mathrm{E}-02$ & $2.0 \mathrm{E}+14$ & $4.8 \mathrm{E}+08$ \\
\hline $\mathrm{I}-128$ & $2.2 \mathrm{E}-07$ & $8.1 \mathrm{E}+08$ & $1.9 \mathrm{E}+03$ \\
\hline I-129 & $5.6 \mathrm{E}-01$ & $2.1 \mathrm{E}+15$ & $4.8 \mathrm{E}+09$ \\
\hline $\mathrm{I}-130$ & $7.1 \mathrm{E}-05$ & $2.6 \mathrm{E}+11$ & $6.1 \mathrm{E}+05$ \\
\hline I-131 & $2.8 \mathrm{E}-02$ & $1.0 \mathrm{E}+14$ & $2.4 \mathrm{E}+08$ \\
\hline I-132 & $1.2 \mathrm{E}-05$ & $4.4 \mathrm{E}+10$ & $1.0 \mathrm{E}+05$ \\
\hline $\mathrm{I}-132 \mathrm{~m}$ & $6.2 \mathrm{E}-06$ & $2.3 E+10$ & $5.4 \mathrm{E}+04$ \\
\hline I-133 & $2.2 \mathrm{E}-04$ & $8.1 \mathrm{E}+11$ & $1.9 \mathrm{E}+06$ \\
\hline I-134 & $6.6 \mathrm{E}-06$ & $2.4 \mathrm{E}+10$ & $5.7 \mathrm{E}+04$ \\
\hline $\mathrm{I}-135$ & $2.5 \mathrm{E}-05$ & $9.3 \mathrm{E}+10$ & $2.2 \mathrm{E}+05$ \\
\hline In-109 & $6.3 \mathrm{E}-06$ & $2.3 \mathrm{E}+10$ & $5.5 \mathrm{E}+04$ \\
\hline In-110a & $4.8 \mathrm{E}-06$ & $1.8 \mathrm{E}+10$ & $4.2 \mathrm{E}+04$ \\
\hline In-110b & $2.7 \mathrm{E}-05$ & $1.0 \mathrm{E}+11$ & $2.3 \mathrm{E}+05$ \\
\hline In-111 & $7.6 \mathrm{E}-05$ & $2.8 \mathrm{E}+11$ & $6.6 \mathrm{E}+05$ \\
\hline In-112 & $3.3 \mathrm{E}-07$ & $1.2 \mathrm{E}+09$ & $2.9 \mathrm{E}+03$ \\
\hline In-113m & $1.0 \mathrm{E}-06$ & $3.7 \mathrm{E}+09$ & $8.7 \mathrm{E}+03$ \\
\hline In-114 & $1.3 \mathrm{E}-09$ & $4.8 \mathrm{E}+06$ & $1.1 \mathrm{E}+01$ \\
\hline In-114m & $3.6 \mathrm{E}-03$ & $1.3 \mathrm{E}+13$ & $3.1 \mathrm{E}+07$ \\
\hline In-115 & $4.0 \mathrm{E}-02$ & $1.5 \mathrm{E}+14$ & $3.5 \mathrm{E}+08$ \\
\hline In-115m & $1.6 \mathrm{E}-06$ & $5.9 \mathrm{E}+09$ & $1.4 \mathrm{E}+04$ \\
\hline In-116m & $6.1 \mathrm{E}-06$ & $2.3 \mathrm{E}+10$ & $5.3 \mathrm{E}+04$ \\
\hline In-117 & $1.6 \mathrm{E}-06$ & $5.9 \mathrm{E}+09$ & $1.4 \mathrm{E}+04$ \\
\hline In- $117 \mathrm{~m}$ & $3.0 \mathrm{E}-05$ & $1.1 \mathrm{E}+11$ & $2.6 \mathrm{E}+05$ \\
\hline In-119 & $1.4 \mathrm{E}-07$ & $5.2 \mathrm{E}+08$ & $1.2 \mathrm{E}+03$ \\
\hline In-119m & $1.2 \mathrm{E}-07$ & $4.4 \mathrm{E}+08$ & $1.0 \mathrm{E}+03$ \\
\hline
\end{tabular}


Table 1. Results of the Screening Analysis

\begin{tabular}{|c|c|c|c|}
\hline Radionuclide & $\begin{array}{c}\text { Level I } \\
\text { Screening Factor } \\
\left(\mathrm{Sv} / \mathrm{Bq}-\mathrm{m}^{-3}\right) \\
\end{array}$ & $\begin{array}{c}\text { Level I } \\
\text { Screening Factor } \\
\left(\mathrm{mrem} / \mathrm{Ci}-\mathrm{m}^{-3}\right) \\
\end{array}$ & $\begin{array}{l}\text { Screening } \\
\text { Dose } \\
\text { mrem/yr } \\
\end{array}$ \\
\hline Ir-182 & $2.5 \mathrm{E}-06$ & $9.3 \mathrm{E}+09$ & $2.2 \mathrm{E}+04$ \\
\hline Ir-184 & $1.1 \mathrm{E}-05$ & $4.1 \mathrm{E}+10$ & $9.5 \mathrm{E}+04$ \\
\hline Ir-185 & $3.3 \mathrm{E}-05$ & $1.2 \mathrm{E}+11$ & $2.9 \mathrm{E}+05$ \\
\hline Ir-186a & $4.8 \mathrm{E}-05$ & $1.8 \mathrm{E}+11$ & $4.2 \mathrm{E}+05$ \\
\hline Ir-186b & $3.8 \mathrm{E}-06$ & $1.4 \mathrm{E}+10$ & $3.3 \mathrm{E}+04$ \\
\hline Ir-187 & 7.3E-06 & $2.7 \mathrm{E}+10$ & $6.3 \mathrm{E}+04$ \\
\hline Ir-188 & $1.3 \mathrm{E}-04$ & $4.8 \mathrm{E}+11$ & $1.1 \mathrm{E}+06$ \\
\hline Ir-189 & $1.5 \mathrm{E}-04$ & $5.6 \mathrm{E}+11$ & $1.3 \mathrm{E}+06$ \\
\hline Ir-190 & $1.1 \mathrm{E}-03$ & $4.1 \mathrm{E}+12$ & $9.5 \mathrm{E}+06$ \\
\hline Ir-190m & 4.4E-06 & $1.6 \mathrm{E}+10$ & $3.8 \mathrm{E}+04$ \\
\hline Ir-190n & 8.7E-07 & $3.2 \mathrm{E}+09$ & $7.5 \mathrm{E}+03$ \\
\hline Ir-191m & $1.8 \mathrm{E}-10$ & $6.7 \mathrm{E}+05$ & $1.6 \mathrm{E}+00$ \\
\hline Ir-192 & $3.3 \mathrm{E}-03$ & $1.2 \mathrm{E}+13$ & $2.9 \mathrm{E}+07$ \\
\hline Ir-192m & $2.4 \mathrm{E}-01$ & $8.9 E+14$ & $2.1 \mathrm{E}+09$ \\
\hline Ir-194 & $2.8 \mathrm{E}-05$ & $1.0 \mathrm{E}+11$ & $2.4 \mathrm{E}+05$ \\
\hline Ir-194m & $1.6 \mathrm{E}-02$ & $5.9 E+13$ & $1.4 \mathrm{E}+08$ \\
\hline Ir-195 & 5.9E-07 & $2.2 \mathrm{E}+09$ & $5.1 \mathrm{E}+03$ \\
\hline Ir-195m & $3.6 \mathrm{E}-06$ & $1.3 \mathrm{E}+10$ & $3.1 \mathrm{E}+04$ \\
\hline $\mathrm{K}-38$ & $2.7 \mathrm{E}-06$ & $1.0 \mathrm{E}+10$ & $2.3 \mathrm{E}+04$ \\
\hline $\mathrm{K}-40$ & $9.4 \mathrm{E}-02$ & $3.5 \mathrm{E}+14$ & $8.1 \mathrm{E}+08$ \\
\hline $\mathrm{K}-42$ & $1.1 \mathrm{E}-05$ & $4.1 \mathrm{E}+10$ & $9.5 \mathrm{E}+04$ \\
\hline $\mathrm{K}-43$ & $4.5 \mathrm{E}-05$ & $1.7 \mathrm{E}+11$ & $3.9 \mathrm{E}+05$ \\
\hline $\mathrm{K}-44$ & $3.7 \mathrm{E}-06$ & $1.4 \mathrm{E}+10$ & $3.2 \mathrm{E}+04$ \\
\hline$K-45$ & $3.0 \mathrm{E}-06$ & $1.1 \mathrm{E}+10$ & $2.6 \mathrm{E}+04$ \\
\hline $\mathrm{Kr}-74$ & $3.2 \mathrm{E}-06$ & $1.2 \mathrm{E}+10$ & $2.8 \mathrm{E}+04$ \\
\hline $\mathrm{Kr}-76$ & $6.8 \mathrm{E}-05$ & $2.5 \mathrm{E}+11$ & $5.9 \mathrm{E}+05$ \\
\hline $\mathrm{Kr}-77$ & $2.7 \mathrm{E}-06$ & $1.0 \mathrm{E}+10$ & $2.3 \mathrm{E}+04$ \\
\hline $\mathrm{Kr}-79$ & $3.1 \mathrm{E}-07$ & $1.1 \mathrm{E}+09$ & $2.7 \mathrm{E}+03$ \\
\hline $\mathrm{Kr}-81$ & $1.3 \mathrm{E}-08$ & $4.8 \mathrm{E}+07$ & $1.1 \mathrm{E}+02$ \\
\hline $\mathrm{Kr}-81 \mathrm{~m}$ & $4.4 \mathrm{E}-19$ & $1.6 \mathrm{E}-03$ & $3.8 \mathrm{E}-09$ \\
\hline $\mathrm{Kr}-83 \mathrm{~m}$ & $1.1 \mathrm{E}-10$ & $4.1 \mathrm{E}+05$ & $9.5 \mathrm{E}-01$ \\
\hline Kr-85 & $2.8 \mathrm{E}-09$ & $1.0 \mathrm{E}+07$ & $2.4 \mathrm{E}+01$ \\
\hline $\mathrm{Kr}-85 \mathrm{~m}$ & $2.0 \mathrm{E}-07$ & $7.4 \mathrm{E}+08$ & $1.7 \mathrm{E}+03$ \\
\hline $\mathrm{Kr}-87$ & $1.0 \mathrm{E}-06$ & $3.7 \mathrm{E}+09$ & $8.7 \mathrm{E}+03$ \\
\hline $\mathrm{Kr}-88$ & $5.4 \mathrm{E}-06$ & $2.0 \mathrm{E}+10$ & $4.7 \mathrm{E}+04$ \\
\hline La-131 & $3.3 \mathrm{E}-06$ & $1.2 \mathrm{E}+10$ & $2.9 \mathrm{E}+04$ \\
\hline La-132 & $1.8 \mathrm{E}-05$ & $6.7 \mathrm{E}+10$ & $1.6 \mathrm{E}+05$ \\
\hline La-134 & 4.8E-07 & $1.8 \mathrm{E}+09$ & $4.2 \mathrm{E}+03$ \\
\hline La-135 & $1.7 \mathrm{E}-06$ & $6.3 \mathrm{E}+09$ & $1.5 \mathrm{E}+04$ \\
\hline La-137 & $6.2 \mathrm{E}-03$ & $2.3 E+13$ & $5.4 \mathrm{E}+07$ \\
\hline La-138 & $2.7 \mathrm{E}-01$ & $1.0 \mathrm{E}+15$ & $2.3 \mathrm{E}+09$ \\
\hline La-140 & $2.4 \mathrm{E}-04$ & $8.9 \mathrm{E}+11$ & $2.1 \mathrm{E}+06$ \\
\hline La-141 & $3.8 \mathrm{E}-06$ & $1.4 \mathrm{E}+10$ & $3.3 \mathrm{E}+04$ \\
\hline La-142 & $9.8 \mathrm{E}-06$ & $3.6 \mathrm{E}+10$ & $8.5 \mathrm{E}+04$ \\
\hline La-143 & $9.6 \mathrm{E}-07$ & $3.6 \mathrm{E}+09$ & $8.3 \mathrm{E}+03$ \\
\hline Lu-169 & $1.1 \mathrm{E}-04$ & $4.1 \mathrm{E}+11$ & $9.5 \mathrm{E}+05$ \\
\hline
\end{tabular}


Table 1. Results of the Screening Analysis

\begin{tabular}{|c|c|c|c|}
\hline Radionuclide & $\begin{array}{c}\text { Level I } \\
\text { Screening Factor } \\
\left(\mathrm{Sv} / \mathrm{Bq}-\mathrm{m}^{-3}\right) \\
\end{array}$ & $\begin{array}{c}\text { Level I } \\
\text { Screening Factor } \\
\left(\mathrm{mrem} / \mathrm{Ci}-\mathrm{m}^{-3}\right) \\
\end{array}$ & $\begin{array}{l}\text { Screening } \\
\text { Dose } \\
\text { mrem/yr } \\
\end{array}$ \\
\hline Lu-170 & $2.3 \mathrm{E}-04$ & $8.5 \mathrm{E}+11$ & $2.0 \mathrm{E}+06$ \\
\hline Lu-171 & $3.9 \mathrm{E}-04$ & $1.4 \mathrm{E}+12$ & $3.4 \mathrm{E}+06$ \\
\hline Lu-172 & 7.3E-04 & $2.7 \mathrm{E}+12$ & $6.3 \mathrm{E}+06$ \\
\hline Lu-173 & $2.8 \mathrm{E}-03$ & $1.0 \mathrm{E}+13$ & $2.4 \mathrm{E}+07$ \\
\hline Lu-174 & $5.6 \mathrm{E}-03$ & $2.1 \mathrm{E}+13$ & $4.8 \mathrm{E}+07$ \\
\hline Lu-174m & $1.4 \mathrm{E}-03$ & $5.2 \mathrm{E}+12$ & $1.2 \mathrm{E}+07$ \\
\hline Lu-176 & $1.3 \mathrm{E}-01$ & $4.8 \mathrm{E}+14$ & $1.1 \mathrm{E}+09$ \\
\hline Lu-176m & $6.4 \mathrm{E}-07$ & $2.4 \mathrm{E}+09$ & $5.5 \mathrm{E}+03$ \\
\hline Lu-177 & $1.5 \mathrm{E}-04$ & $5.6 \mathrm{E}+11$ & $1.3 \mathrm{E}+06$ \\
\hline Lu-177m & 7.8E-03 & $2.9 \mathrm{E}+13$ & $6.7 \mathrm{E}+07$ \\
\hline Lu-178 & $3.2 \mathrm{E}-07$ & $1.2 \mathrm{E}+09$ & $2.8 \mathrm{E}+03$ \\
\hline Lu-178m & $1.8 \mathrm{E}-06$ & $6.7 \mathrm{E}+09$ & $1.6 \mathrm{E}+04$ \\
\hline Lu-179 & 9.8E-07 & $3.6 \mathrm{E}+09$ & $8.5 \mathrm{E}+03$ \\
\hline Md-257 & $9.5 \mathrm{E}-05$ & $3.5 \mathrm{E}+11$ & $8.2 \mathrm{E}+05$ \\
\hline Md-258 & $3.5 \mathrm{E}-02$ & $1.3 \mathrm{E}+14$ & $3.0 \mathrm{E}+08$ \\
\hline Mg-28 & $1.8 \mathrm{E}-04$ & $6.7 \mathrm{E}+11$ & $1.6 \mathrm{E}+06$ \\
\hline $\mathrm{Mn}-51$ & $2.6 \mathrm{E}-06$ & $9.6 \mathrm{E}+09$ & $2.2 \mathrm{E}+04$ \\
\hline $\mathrm{Mn}-52$ & $9.6 \mathrm{E}-04$ & $3.6 \mathrm{E}+12$ & $8.3 \mathrm{E}+06$ \\
\hline $\mathrm{Mn}-52 \mathrm{~m}$ & $3.7 \mathrm{E}-06$ & $1.4 \mathrm{E}+10$ & $3.2 \mathrm{E}+04$ \\
\hline Mn-53 & $1.8 \mathrm{E}-04$ & $6.7 \mathrm{E}+11$ & $1.6 \mathrm{E}+06$ \\
\hline $\mathrm{Mn}-54$ & $9.7 \mathrm{E}-03$ & $3.6 \mathrm{E}+13$ & $8.4 \mathrm{E}+07$ \\
\hline Mn-56 & $9.1 \mathrm{E}-06$ & $3.4 \mathrm{E}+10$ & $7.9 \mathrm{E}+04$ \\
\hline Mo-101 & $2.2 \mathrm{E}-06$ & $8.1 \mathrm{E}+09$ & $1.9 \mathrm{E}+04$ \\
\hline Мo-90 & 4.7E-05 & $1.7 \mathrm{E}+11$ & $4.1 \mathrm{E}+05$ \\
\hline Мo-93 & $2.1 \mathrm{E}-03$ & $7.8 \mathrm{E}+12$ & $1.8 \mathrm{E}+07$ \\
\hline Mo-93m & $2.5 \mathrm{E}-05$ & $9.3 \mathrm{E}+10$ & $2.2 \mathrm{E}+05$ \\
\hline Мo-99 & 2.3E-04 & $8.5 E+11$ & $2.0 \mathrm{E}+06$ \\
\hline $\mathrm{N}-13$ & 9.7E-07 & $3.6 \mathrm{E}+09$ & $8.4 \mathrm{E}+03$ \\
\hline $\mathrm{Na}-22$ & $1.0 \mathrm{E}-01$ & $3.7 \mathrm{E}+14$ & $8.7 \mathrm{E}+08$ \\
\hline $\mathrm{Na}-24$ & $1.1 \mathrm{E}-04$ & $4.1 \mathrm{E}+11$ & $9.5 \mathrm{E}+05$ \\
\hline $\mathrm{Nb}-88$ & $6.1 \mathrm{E}-06$ & $2.3 \mathrm{E}+10$ & $5.3 \mathrm{E}+04$ \\
\hline $\mathrm{Nb}-89 \mathrm{a}$ & 7.2E-06 & $2.7 \mathrm{E}+10$ & $6.2 \mathrm{E}+04$ \\
\hline $\mathrm{Nb}-89 \mathrm{~b}$ & $1.5 \mathrm{E}-05$ & $5.6 \mathrm{E}+10$ & $1.3 \mathrm{E}+05$ \\
\hline $\mathrm{Nb}-90$ & $1.0 \mathrm{E}-04$ & $3.7 \mathrm{E}+11$ & $8.7 \mathrm{E}+05$ \\
\hline $\mathrm{Nb}-93 \mathrm{~m}$ & $3.2 \mathrm{E}-04$ & $1.2 \mathrm{E}+12$ & $2.8 \mathrm{E}+06$ \\
\hline $\mathrm{Nb}-94$ & $3.8 \mathrm{E}-01$ & $1.4 \mathrm{E}+15$ & $3.3 \mathrm{E}+09$ \\
\hline $\mathrm{Nb}-95$ & 1.2E-03 & $4.4 \mathrm{E}+12$ & $1.0 \mathrm{E}+07$ \\
\hline $\mathrm{Nb}-95 \mathrm{~m}$ & $2.1 \mathrm{E}-04$ & $7.8 \mathrm{E}+11$ & $1.8 \mathrm{E}+06$ \\
\hline $\mathrm{Nb}-96$ & $1.1 \mathrm{E}-04$ & $4.1 \mathrm{E}+11$ & $9.5 \mathrm{E}+05$ \\
\hline Nb-97 & 2.1E-06 & $7.8 \mathrm{E}+09$ & $1.8 \mathrm{E}+04$ \\
\hline $\mathrm{Nb}-97 \mathrm{~m}$ & $5.1 \mathrm{E}-08$ & $1.9 \mathrm{E}+08$ & $4.4 \mathrm{E}+02$ \\
\hline Nb-98 & $6.1 \mathrm{E}-06$ & $2.3 \mathrm{E}+10$ & $5.3 \mathrm{E}+04$ \\
\hline Nd-136 & 4.5E-06 & $1.7 \mathrm{E}+10$ & $3.9 \mathrm{E}+04$ \\
\hline Nd-138 & 9.9E-06 & $3.7 \mathrm{E}+10$ & $8.6 \mathrm{E}+04$ \\
\hline Nd-139 & $1.0 \mathrm{E}-06$ & $3.7 \mathrm{E}+09$ & $8.7 \mathrm{E}+03$ \\
\hline Nd-139m & $1.9 \mathrm{E}-05$ & $7.0 \mathrm{E}+10$ & $1.6 \mathrm{E}+05$ \\
\hline
\end{tabular}


Table 1. Results of the Screening Analysis

\begin{tabular}{|c|c|c|c|}
\hline Radionuclide & $\begin{array}{c}\text { Level I } \\
\text { Screening Factor } \\
\left(\mathrm{Sv} / \mathrm{Bq}-\mathrm{m}^{-3}\right) \\
\end{array}$ & $\begin{array}{c}\text { Level I } \\
\text { Screening Factor } \\
\left(\mathrm{mrem} / \mathrm{Ci}-\mathrm{m}^{-3}\right) \\
\end{array}$ & $\begin{array}{l}\text { Screening } \\
\text { Dose } \\
\text { mrem/yr } \\
\end{array}$ \\
\hline Nd-141 & $3.8 \mathrm{E}-07$ & $1.4 \mathrm{E}+09$ & $3.3 \mathrm{E}+03$ \\
\hline $\mathrm{Nd}-141 \mathrm{~m}$ & $2.7 \mathrm{E}-08$ & $1.0 \mathrm{E}+08$ & $2.3 \mathrm{E}+02$ \\
\hline Nd-147 & 4.7E-04 & $1.7 \mathrm{E}+12$ & $4.1 \mathrm{E}+06$ \\
\hline Nd-149 & $4.4 \mathrm{E}-06$ & $1.6 \mathrm{E}+10$ & $3.8 \mathrm{E}+04$ \\
\hline $\mathrm{Nd}-151$ & $1.4 \mathrm{E}-06$ & $5.2 \mathrm{E}+09$ & $1.2 \mathrm{E}+04$ \\
\hline $\mathrm{Ne}-19$ & $2.3 \mathrm{E}-15$ & $8.5 \mathrm{E}+00$ & $2.0 \mathrm{E}-05$ \\
\hline Ni-56 & $2.5 \mathrm{E}-03$ & $9.3 \mathrm{E}+12$ & $2.2 \mathrm{E}+07$ \\
\hline $\mathrm{Ni}-57$ & $3.1 \mathrm{E}-04$ & $1.1 \mathrm{E}+12$ & $2.7 \mathrm{E}+06$ \\
\hline $\mathrm{Ni}-59$ & $6.8 \mathrm{E}-04$ & $2.5 \mathrm{E}+12$ & $5.9 \mathrm{E}+06$ \\
\hline Ni-63 & $1.6 \mathrm{E}-03$ & $5.9 \mathrm{E}+12$ & $1.4 \mathrm{E}+07$ \\
\hline $\mathrm{Ni}-65$ & $3.3 \mathrm{E}-06$ & $1.2 \mathrm{E}+10$ & $2.9 \mathrm{E}+04$ \\
\hline Ni-66 & $1.6 \mathrm{E}-03$ & $5.9 \mathrm{E}+12$ & $1.4 \mathrm{E}+07$ \\
\hline Np-232 & $2.8 \mathrm{E}-06$ & $1.0 \mathrm{E}+10$ & $2.4 \mathrm{E}+04$ \\
\hline $\mathrm{Np}-233$ & $1.9 \mathrm{E}-07$ & $7.0 \mathrm{E}+08$ & $1.6 \mathrm{E}+03$ \\
\hline $\mathrm{Np}-234$ & $3.0 \mathrm{E}-04$ & $1.1 \mathrm{E}+12$ & $2.6 \mathrm{E}+06$ \\
\hline $\mathrm{Np}-235$ & $1.2 \mathrm{E}-04$ & $4.4 \mathrm{E}+11$ & $1.0 \mathrm{E}+06$ \\
\hline $\mathrm{Np}-236 \mathrm{a}$ & $3.8 \mathrm{E}+03$ & $1.4 \mathrm{E}+19$ & $3.3 \mathrm{E}+13$ \\
\hline $\mathrm{Np}-236 \mathrm{~b}$ & 2.3E-04 & $8.5 \mathrm{E}+11$ & $2.0 \mathrm{E}+06$ \\
\hline Np-237 & $1.3 \mathrm{E}+00$ & $4.8 \mathrm{E}+15$ & $1.1 \mathrm{E}+10$ \\
\hline $\mathrm{Np}-238$ & $1.7 \mathrm{E}-04$ & $6.3 \mathrm{E}+11$ & $1.5 \mathrm{E}+06$ \\
\hline $\mathrm{Np}-239$ & $8.5 \mathrm{E}-05$ & $3.1 \mathrm{E}+11$ & $7.4 \mathrm{E}+05$ \\
\hline $\mathrm{Np}-240$ & $3.4 \mathrm{E}-06$ & $1.3 \mathrm{E}+10$ & $2.9 \mathrm{E}+04$ \\
\hline $\mathrm{Np}-240 \mathrm{~m}$ & $2.6 \mathrm{E}-07$ & $9.6 \mathrm{E}+08$ & $2.2 \mathrm{E}+03$ \\
\hline $\mathrm{O}-15$ & $1.3 \mathrm{E}-07$ & $4.8 \mathrm{E}+08$ & $1.1 \mathrm{E}+03$ \\
\hline Os-180 & $1.9 \mathrm{E}-06$ & $7.0 \mathrm{E}+09$ & $1.6 \mathrm{E}+04$ \\
\hline Os-181 & 7.9E-06 & $2.9 \mathrm{E}+10$ & $6.8 \mathrm{E}+04$ \\
\hline Os-182 & $6.9 \mathrm{E}-05$ & $2.6 \mathrm{E}+11$ & $6.0 \mathrm{E}+05$ \\
\hline Os-185 & $2.8 \mathrm{E}-03$ & $1.0 \mathrm{E}+13$ & $2.4 \mathrm{E}+07$ \\
\hline Os-189m & 7.3E-08 & $2.7 \mathrm{E}+08$ & $6.3 \mathrm{E}+02$ \\
\hline Os-190m & $1.5 \mathrm{E}-06$ & $5.6 \mathrm{E}+09$ & $1.3 \mathrm{E}+04$ \\
\hline Os-191 & $3.1 \mathrm{E}-04$ & $1.1 \mathrm{E}+12$ & $2.7 \mathrm{E}+06$ \\
\hline Os-191m & $1.2 \mathrm{E}-05$ & $4.4 \mathrm{E}+10$ & $1.0 \mathrm{E}+05$ \\
\hline Os-193 & $3.5 \mathrm{E}-05$ & $1.3 \mathrm{E}+11$ & $3.0 \mathrm{E}+05$ \\
\hline Os-194 & $1.2 \mathrm{E}-02$ & $4.4 \mathrm{E}+13$ & $1.0 \mathrm{E}+08$ \\
\hline P-30 & $1.9 \mathrm{E}-07$ & $7.0 \mathrm{E}+08$ & $1.6 \mathrm{E}+03$ \\
\hline P-32 & 7.3E-03 & $2.7 \mathrm{E}+13$ & $6.3 \mathrm{E}+07$ \\
\hline P-33 & 9.8E-04 & $3.6 \mathrm{E}+12$ & $8.5 \mathrm{E}+06$ \\
\hline $\mathrm{Pa}-227$ & $9.8 \mathrm{E}-05$ & $3.6 \mathrm{E}+11$ & $8.5 \mathrm{E}+05$ \\
\hline $\mathrm{Pa}-228$ & $1.1 \mathrm{E}-03$ & $4.1 \mathrm{E}+12$ & $9.5 \mathrm{E}+06$ \\
\hline $\mathrm{Pa}-230$ & $5.4 \mathrm{E}-03$ & $2.0 \mathrm{E}+13$ & $4.7 \mathrm{E}+07$ \\
\hline $\mathrm{Pa}-231$ & $3.0 \mathrm{E}+00$ & $1.1 \mathrm{E}+16$ & $2.6 \mathrm{E}+10$ \\
\hline $\mathrm{Pa}-232$ & $2.0 \mathrm{E}-04$ & $7.4 \mathrm{E}+11$ & $1.7 \mathrm{E}+06$ \\
\hline $\mathrm{Pa}-233$ & 6.7E-04 & $2.5 \mathrm{E}+12$ & $5.8 \mathrm{E}+06$ \\
\hline $\mathrm{Pa}-234$ & $2.5 \mathrm{E}-05$ & $9.3 \mathrm{E}+10$ & $2.2 \mathrm{E}+05$ \\
\hline $\mathrm{Pa}-234 \mathrm{~m}$ & $5.3 \mathrm{E}-10$ & $2.0 \mathrm{E}+06$ & $4.6 \mathrm{E}+00$ \\
\hline $\mathrm{Pb}-195 \mathrm{~m}$ & $2.9 \mathrm{E}-06$ & $1.1 \mathrm{E}+10$ & $2.5 \mathrm{E}+04$ \\
\hline
\end{tabular}


Table 1. Results of the Screening Analysis

\begin{tabular}{|c|c|c|c|}
\hline Radionuclide & $\begin{array}{c}\text { Level I } \\
\text { Screening Factor } \\
\left(\mathrm{Sv} / \mathrm{Bq}-\mathrm{m}^{-3}\right) \\
\end{array}$ & $\begin{array}{c}\text { Level I } \\
\text { Screening Factor } \\
\left(\mathrm{mrem} / \mathrm{Ci}-\mathrm{m}^{-3}\right) \\
\end{array}$ & $\begin{array}{l}\text { Screening } \\
\text { Dose } \\
\text { mrem/yr } \\
\end{array}$ \\
\hline $\mathrm{Pb}-198$ & $9.3 \mathrm{E}-06$ & $3.4 \mathrm{E}+10$ & $8.0 \mathrm{E}+04$ \\
\hline $\mathrm{Pb}-199$ & $5.8 \mathrm{E}-06$ & $2.1 \mathrm{E}+10$ & $5.0 \mathrm{E}+04$ \\
\hline $\mathrm{Pb}-200$ & $6.2 \mathrm{E}-05$ & $2.3 \mathrm{E}+11$ & $5.4 \mathrm{E}+05$ \\
\hline $\mathrm{Pb}-201$ & $1.6 \mathrm{E}-05$ & $5.9 \mathrm{E}+10$ & $1.4 \mathrm{E}+05$ \\
\hline $\mathrm{Pb}-202$ & $1.3 \mathrm{E}-01$ & $4.8 \mathrm{E}+14$ & $1.1 \mathrm{E}+09$ \\
\hline $\mathrm{Pb}-202 \mathrm{~m}$ & $1.5 \mathrm{E}-05$ & $5.6 \mathrm{E}+10$ & $1.3 \mathrm{E}+05$ \\
\hline $\mathrm{Pb}-203$ & $4.4 \mathrm{E}-05$ & $1.6 \mathrm{E}+11$ & $3.8 \mathrm{E}+05$ \\
\hline $\mathrm{Pb}-205$ & $5.0 \mathrm{E}-04$ & $1.9 \mathrm{E}+12$ & $4.3 \mathrm{E}+06$ \\
\hline $\mathrm{Pb}-209$ & $1.8 \mathrm{E}-07$ & $6.7 \mathrm{E}+08$ & $1.6 \mathrm{E}+03$ \\
\hline $\mathrm{Pb}-210$ & 7.1E-01 & $2.6 \mathrm{E}+15$ & $6.1 \mathrm{E}+09$ \\
\hline $\mathrm{Pb}-211$ & $1.6 \mathrm{E}-05$ & $5.9 \mathrm{E}+10$ & $1.4 \mathrm{E}+05$ \\
\hline $\mathrm{Pb}-212$ & $3.4 \mathrm{E}-04$ & $1.3 \mathrm{E}+12$ & $2.9 \mathrm{E}+06$ \\
\hline $\mathrm{Pb}-214$ & $2.0 \mathrm{E}-05$ & $7.4 \mathrm{E}+10$ & $1.7 \mathrm{E}+05$ \\
\hline Pd-100 & $5.2 \mathrm{E}-04$ & $1.9 \mathrm{E}+12$ & $4.5 \mathrm{E}+06$ \\
\hline Pd-101 & $1.2 \mathrm{E}-05$ & $4.4 \mathrm{E}+10$ & $1.0 \mathrm{E}+05$ \\
\hline Pd-103 & $9.3 \mathrm{E}-05$ & $3.4 \mathrm{E}+11$ & $8.0 \mathrm{E}+05$ \\
\hline Pd-107 & $1.3 \mathrm{E}-04$ & $4.8 \mathrm{E}+11$ & $1.1 \mathrm{E}+06$ \\
\hline Pd-109 & $6.1 \mathrm{E}-06$ & $2.3 \mathrm{E}+10$ & $5.3 \mathrm{E}+04$ \\
\hline Pm-141 & $1.2 \mathrm{E}-06$ & $4.4 \mathrm{E}+09$ & $1.0 \mathrm{E}+04$ \\
\hline Pm-142 & $1.5 \mathrm{E}-08$ & $5.6 \mathrm{E}+07$ & $1.3 \mathrm{E}+02$ \\
\hline Pm-143 & $3.1 \mathrm{E}-03$ & $1.1 \mathrm{E}+13$ & $2.7 \mathrm{E}+07$ \\
\hline Pm-144 & $2.1 \mathrm{E}-02$ & $7.8 \mathrm{E}+13$ & $1.8 \mathrm{E}+08$ \\
\hline Pm-145 & $4.4 \mathrm{E}-03$ & $1.6 \mathrm{E}+13$ & $3.8 \mathrm{E}+07$ \\
\hline Pm-146 & $5.1 \mathrm{E}-02$ & $1.9 \mathrm{E}+14$ & $4.4 \mathrm{E}+08$ \\
\hline Pm-147 & $3.2 \mathrm{E}-04$ & $1.2 \mathrm{E}+12$ & $2.8 \mathrm{E}+06$ \\
\hline Pm-148 & $6.8 \mathrm{E}-04$ & $2.5 \mathrm{E}+12$ & $5.9 \mathrm{E}+06$ \\
\hline Pm-148m & 4.1E-03 & $1.5 \mathrm{E}+13$ & $3.5 \mathrm{E}+07$ \\
\hline Pm-149 & $8.3 \mathrm{E}-05$ & $3.1 \mathrm{E}+11$ & $7.2 \mathrm{E}+05$ \\
\hline Pm-150 & 8.1E-06 & $3.0 \mathrm{E}+10$ & $7.0 \mathrm{E}+04$ \\
\hline Pm-151 & 4.1E-05 & $1.5 \mathrm{E}+11$ & $3.5 \mathrm{E}+05$ \\
\hline Po-203 & $6.2 \mathrm{E}-06$ & $2.3 \mathrm{E}+10$ & $5.4 \mathrm{E}+04$ \\
\hline Po-205 & $1.3 \mathrm{E}-05$ & $4.8 \mathrm{E}+10$ & $1.1 \mathrm{E}+05$ \\
\hline Po-207 & $1.9 \mathrm{E}-05$ & $7.0 \mathrm{E}+10$ & $1.6 \mathrm{E}+05$ \\
\hline Po-210 & $1.6 \mathrm{E}-01$ & $5.9 \mathrm{E}+14$ & $1.4 \mathrm{E}+09$ \\
\hline Po-211 & $1.7 \mathrm{E}-12$ & $6.3 \mathrm{E}+03$ & $1.5 \mathrm{E}-02$ \\
\hline Po-212 & $0.0 \mathrm{E}+00$ & $0.0 \mathrm{E}+00$ & $0.0 \mathrm{E}+00$ \\
\hline Po-213 & $6.4 \mathrm{E}-17$ & $2.4 \mathrm{E}-01$ & $5.5 \mathrm{E}-07$ \\
\hline Po-214 & $5.7 \mathrm{E}-18$ & $2.1 \mathrm{E}-02$ & 4.9E-08 \\
\hline Po-215 & $1.3 \mathrm{E}-11$ & $4.8 \mathrm{E}+04$ & $1.1 \mathrm{E}-01$ \\
\hline Po-216 & 1.3E-09 & $4.8 \mathrm{E}+06$ & $1.1 \mathrm{E}+01$ \\
\hline Po-218 & $2.0 \mathrm{E}-06$ & $7.4 \mathrm{E}+09$ & $1.7 \mathrm{E}+04$ \\
\hline Pr-136 & $2.5 \mathrm{E}-06$ & $9.3 \mathrm{E}+09$ & $2.2 \mathrm{E}+04$ \\
\hline Pr-137 & $1.7 \mathrm{E}-06$ & $6.3 \mathrm{E}+09$ & $1.5 \mathrm{E}+04$ \\
\hline Pr-138 & 4.9E-08 & $1.8 \mathrm{E}+08$ & $4.2 \mathrm{E}+02$ \\
\hline Pr-138m & $1.1 \mathrm{E}-05$ & $4.1 \mathrm{E}+10$ & $9.5 \mathrm{E}+04$ \\
\hline Pr-139 & $2.5 \mathrm{E}-06$ & $9.3 E+09$ & $2.2 \mathrm{E}+04$ \\
\hline
\end{tabular}


Table 1. Results of the Screening Analysis

\begin{tabular}{|c|c|c|c|}
\hline Radionuclide & $\begin{array}{c}\text { Level I } \\
\text { Screening Factor } \\
\left(\mathrm{Sv} / \mathrm{Bq}-\mathrm{m}^{-3}\right) \\
\end{array}$ & $\begin{array}{c}\text { Level I } \\
\text { Screening Factor } \\
\left(\mathrm{mrem} / \mathrm{Ci}-\mathrm{m}^{-3}\right) \\
\end{array}$ & $\begin{array}{l}\text { Screening } \\
\text { Dose } \\
\text { mrem/yr } \\
\end{array}$ \\
\hline Pr-142 & 2.7E-05 & $1.0 \mathrm{E}+11$ & $2.3 \mathrm{E}+05$ \\
\hline $\operatorname{Pr}-142 \mathrm{~m}$ & $3.5 \mathrm{E}-07$ & $1.3 \mathrm{E}+09$ & $3.0 \mathrm{E}+03$ \\
\hline Pr-143 & $5.2 \mathrm{E}-04$ & $1.9 \mathrm{E}+12$ & $4.5 \mathrm{E}+06$ \\
\hline $\operatorname{Pr}-144$ & $1.1 \mathrm{E}-07$ & $4.1 \mathrm{E}+08$ & $9.5 \mathrm{E}+02$ \\
\hline $\operatorname{Pr}-144 m$ & $3.7 \mathrm{E}-08$ & $1.4 \mathrm{E}+08$ & $3.2 \mathrm{E}+02$ \\
\hline Pr-145 & $1.7 \mathrm{E}-06$ & $6.3 \mathrm{E}+09$ & $1.5 \mathrm{E}+04$ \\
\hline $\operatorname{Pr}-147$ & $1.4 \mathrm{E}-06$ & $5.2 \mathrm{E}+09$ & $1.2 \mathrm{E}+04$ \\
\hline Pt-186 & $6.4 \mathrm{E}-06$ & $2.4 \mathrm{E}+10$ & $5.5 \mathrm{E}+04$ \\
\hline Pt-188 & $9.6 \mathrm{E}-04$ & $3.6 \mathrm{E}+12$ & $8.3 \mathrm{E}+06$ \\
\hline Pt-189 & $1.2 \mathrm{E}-05$ & $4.4 \mathrm{E}+10$ & $1.0 \mathrm{E}+05$ \\
\hline Pt-191 & $6.4 \mathrm{E}-05$ & $2.4 \mathrm{E}+11$ & $5.5 \mathrm{E}+05$ \\
\hline Pt-193 & $1.7 \mathrm{E}-04$ & $6.3 E+11$ & $1.5 \mathrm{E}+06$ \\
\hline Pt-193m & $7.6 \mathrm{E}-05$ & $2.8 \mathrm{E}+11$ & $6.6 \mathrm{E}+05$ \\
\hline Pt-195m & $1.1 \mathrm{E}-04$ & $4.1 \mathrm{E}+11$ & $9.5 \mathrm{E}+05$ \\
\hline Pt-197 & $7.3 \mathrm{E}-06$ & $2.7 \mathrm{E}+10$ & $6.3 \mathrm{E}+04$ \\
\hline Pt-197m & $1.0 \mathrm{E}-06$ & $3.7 \mathrm{E}+09$ & $8.7 \mathrm{E}+03$ \\
\hline Pt-199 & $9.1 \mathrm{E}-07$ & $3.4 \mathrm{E}+09$ & $7.9 \mathrm{E}+03$ \\
\hline Pt-200 & $1.6 \mathrm{E}-05$ & $5.9 \mathrm{E}+10$ & $1.4 \mathrm{E}+05$ \\
\hline $\mathrm{Pu}-234$ & $1.2 \mathrm{E}-04$ & $4.4 \mathrm{E}+11$ & $1.0 \mathrm{E}+06$ \\
\hline $\mathrm{Pu}-235$ & $1.6 \mathrm{E}-07$ & $5.9 \mathrm{E}+08$ & $1.4 \mathrm{E}+03$ \\
\hline $\mathrm{Pu}-236$ & $3.5 \mathrm{E}-01$ & $1.3 \mathrm{E}+15$ & $3.0 \mathrm{E}+09$ \\
\hline $\mathrm{Pu}-237$ & $1.6 \mathrm{E}-04$ & $5.9 \mathrm{E}+11$ & $1.4 \mathrm{E}+06$ \\
\hline $\mathrm{Pu}-238$ & 8.9E-01 & $3.3 E+15$ & $7.7 E+09$ \\
\hline $\mathrm{Pu}-239$ & $1.0 \mathrm{E}+00$ & $3.7 \mathrm{E}+15$ & $8.7 E+09$ \\
\hline $\mathrm{Pu}-240$ & $1.0 \mathrm{E}+00$ & $3.7 \mathrm{E}+15$ & $8.7 E+09$ \\
\hline $\mathrm{Pu}-241$ & $2.0 \mathrm{E}-02$ & $7.4 \mathrm{E}+13$ & $1.7 \mathrm{E}+08$ \\
\hline $\mathrm{Pu}-242$ & $9.5 \mathrm{E}-01$ & $3.5 E+15$ & $8.2 \mathrm{E}+09$ \\
\hline $\mathrm{Pu}-243$ & $6.2 \mathrm{E}-07$ & $2.3 \mathrm{E}+09$ & $5.4 \mathrm{E}+03$ \\
\hline $\mathrm{Pu}-244$ & $1.0 \mathrm{E}+00$ & $3.7 \mathrm{E}+15$ & $8.7 \mathrm{E}+09$ \\
\hline $\mathrm{Pu}-245$ & $1.3 \mathrm{E}-05$ & $4.8 \mathrm{E}+10$ & $1.1 \mathrm{E}+05$ \\
\hline $\mathrm{Pu}-246$ & $1.5 \mathrm{E}-03$ & $5.6 \mathrm{E}+12$ & $1.3 \mathrm{E}+07$ \\
\hline Ra-222 & 4.7E-08 & $1.7 \mathrm{E}+08$ & $4.1 \mathrm{E}+02$ \\
\hline $\mathrm{Ra}-223$ & $5.6 \mathrm{E}-02$ & $2.1 \mathrm{E}+14$ & $4.8 \mathrm{E}+08$ \\
\hline Ra-224 & $1.6 \mathrm{E}-02$ & $5.9 E+13$ & $1.4 \mathrm{E}+08$ \\
\hline $\mathrm{Ra}-225$ & 4.7E-02 & $1.7 \mathrm{E}+14$ & $4.1 \mathrm{E}+08$ \\
\hline Ra-226 & 8.3E-01 & $3.1 \mathrm{E}+15$ & $7.2 \mathrm{E}+09$ \\
\hline Ra-227 & $1.1 \mathrm{E}-05$ & $4.1 \mathrm{E}+10$ & $9.5 \mathrm{E}+04$ \\
\hline Ra-228 & 4.2E-01 & $1.6 \mathrm{E}+15$ & $3.6 \mathrm{E}+09$ \\
\hline Rb-79 & $2.4 \mathrm{E}-06$ & $8.9 \mathrm{E}+09$ & $2.1 \mathrm{E}+04$ \\
\hline $\mathrm{Rb}-80$ & $1.8 \mathrm{E}-08$ & $6.7 \mathrm{E}+07$ & $1.6 \mathrm{E}+02$ \\
\hline $\mathrm{Rb}-81$ & $5.4 \mathrm{E}-06$ & $2.0 \mathrm{E}+10$ & $4.7 \mathrm{E}+04$ \\
\hline $\mathrm{Rb}-81 \mathrm{~m}$ & 5.7E-07 & $2.1 \mathrm{E}+09$ & $4.9 \mathrm{E}+03$ \\
\hline $\mathrm{Rb}-82$ & 5.3E-08 & $2.0 \mathrm{E}+08$ & $4.6 \mathrm{E}+02$ \\
\hline $\mathrm{Rb}-82 \mathrm{~m}$ & $3.1 \mathrm{E}-05$ & $1.1 \mathrm{E}+11$ & $2.7 \mathrm{E}+05$ \\
\hline $\mathrm{Rb}-83$ & 7.5E-03 & $2.8 \mathrm{E}+13$ & $6.5 \mathrm{E}+07$ \\
\hline $\mathrm{Rb}-84$ & 7.3E-03 & $2.7 \mathrm{E}+13$ & $6.3 \mathrm{E}+07$ \\
\hline
\end{tabular}


Table 1. Results of the Screening Analysis

\begin{tabular}{|c|c|c|c|}
\hline Radionuclide & $\begin{array}{c}\text { Level I } \\
\text { Screening Factor } \\
\left(\mathrm{Sv} / \mathrm{Bq}-\mathrm{m}^{-3}\right) \\
\end{array}$ & $\begin{array}{c}\text { Level I } \\
\text { Screening Factor } \\
\left(\mathrm{mrem} / \mathrm{Ci}-\mathrm{m}^{-3}\right) \\
\end{array}$ & $\begin{array}{l}\text { Screening } \\
\text { Dose } \\
\text { mrem/yr } \\
\end{array}$ \\
\hline Rb-86 & $4.8 \mathrm{E}-03$ & $1.8 \mathrm{E}+13$ & $4.2 \mathrm{E}+07$ \\
\hline $\mathrm{Rb}-87$ & $1.6 \mathrm{E}-02$ & $5.9 \mathrm{E}+13$ & $1.4 \mathrm{E}+08$ \\
\hline $\mathrm{Rb}-88$ & $1.0 \mathrm{E}-06$ & $3.7 \mathrm{E}+09$ & $8.7 E+03$ \\
\hline $\mathrm{Rb}-89$ & $3.4 \mathrm{E}-06$ & $1.3 \mathrm{E}+10$ & $2.9 \mathrm{E}+04$ \\
\hline Re-177 & $1.2 \mathrm{E}-06$ & $4.4 \mathrm{E}+09$ & $1.0 \mathrm{E}+04$ \\
\hline Re-178 & $1.7 \mathrm{E}-06$ & $6.3 \mathrm{E}+09$ & $1.5 \mathrm{E}+04$ \\
\hline Re-180 & $2.2 \mathrm{E}-07$ & $8.1 \mathrm{E}+08$ & $1.9 \mathrm{E}+03$ \\
\hline $\operatorname{Re}-181$ & $3.6 \mathrm{E}-05$ & $1.3 \mathrm{E}+11$ & $3.1 \mathrm{E}+05$ \\
\hline $\operatorname{Re}-182 \mathrm{a}$ & $2.5 \mathrm{E}-05$ & $9.3 \mathrm{E}+10$ & $2.2 \mathrm{E}+05$ \\
\hline Re-182b & $2.8 \mathrm{E}-04$ & $1.0 \mathrm{E}+12$ & $2.4 \mathrm{E}+06$ \\
\hline Re-184 & $1.9 \mathrm{E}-03$ & $7.0 \mathrm{E}+12$ & $1.6 \mathrm{E}+07$ \\
\hline $\operatorname{Re}-184 m$ & $7.9 \mathrm{E}-03$ & $2.9 \mathrm{E}+13$ & $6.8 \mathrm{E}+07$ \\
\hline $\operatorname{Re}-186$ & $2.4 \mathrm{E}-04$ & $8.9 \mathrm{E}+11$ & $2.1 \mathrm{E}+06$ \\
\hline Re-186m & $2.1 \mathrm{E}-02$ & $7.8 \mathrm{E}+13$ & $1.8 \mathrm{E}+08$ \\
\hline Re-187 & $1.8 \mathrm{E}-05$ & $6.7 E+10$ & $1.6 \mathrm{E}+05$ \\
\hline Re-188 & $2.3 \mathrm{E}-05$ & $8.5 E+10$ & $2.0 \mathrm{E}+05$ \\
\hline Re-188m & $5.4 \mathrm{E}-07$ & $2.0 \mathrm{E}+09$ & $4.7 E+03$ \\
\hline Re-189 & $2.4 \mathrm{E}-05$ & $8.9 \mathrm{E}+10$ & $2.1 \mathrm{E}+05$ \\
\hline Rh-99 & 5.9E-04 & $2.2 \mathrm{E}+12$ & $5.1 \mathrm{E}+06$ \\
\hline Rh-99m & $5.9 \mathrm{E}-06$ & $2.2 \mathrm{E}+10$ & $5.1 \mathrm{E}+04$ \\
\hline Rh-100 & $9.8 \mathrm{E}-05$ & $3.6 \mathrm{E}+11$ & $8.5 \mathrm{E}+05$ \\
\hline Rh-101 & $1.3 \mathrm{E}-02$ & $4.8 E+13$ & $1.1 \mathrm{E}+08$ \\
\hline $\mathrm{Rh}-101 \mathrm{~m}$ & $9.1 \mathrm{E}-05$ & $3.4 \mathrm{E}+11$ & $7.9 \mathrm{E}+05$ \\
\hline Rh-102 & $8.0 \mathrm{E}-02$ & $3.0 \mathrm{E}+14$ & $6.9 \mathrm{E}+08$ \\
\hline $\mathrm{Rh}-102 \mathrm{~m}$ & $5.5 \mathrm{E}-03$ & $2.0 \mathrm{E}+13$ & $4.8 \mathrm{E}+07$ \\
\hline $\mathrm{Rh}-103 \mathrm{~m}$ & $1.2 \mathrm{E}-08$ & $4.4 \mathrm{E}+07$ & $1.0 \mathrm{E}+02$ \\
\hline $\mathrm{Rh}-105$ & $2.4 \mathrm{E}-05$ & $8.9 \mathrm{E}+10$ & $2.1 \mathrm{E}+05$ \\
\hline Rh-106 & 2.7E-09 & $1.0 \mathrm{E}+07$ & $2.3 \mathrm{E}+01$ \\
\hline Rh-106m & $1.3 \mathrm{E}-05$ & $4.8 \mathrm{E}+10$ & $1.1 \mathrm{E}+05$ \\
\hline Rh-107 & $5.2 \mathrm{E}-07$ & $1.9 \mathrm{E}+09$ & $4.5 \mathrm{E}+03$ \\
\hline Rn-218 & $1.2 \mathrm{E}-15$ & $4.4 \mathrm{E}+00$ & $1.0 \mathrm{E}-05$ \\
\hline Rn-219 & 2.9E-08 & $1.1 \mathrm{E}+08$ & $2.5 \mathrm{E}+02$ \\
\hline $\mathrm{Rn}-220$ & $5.0 \mathrm{E}-07$ & $1.9 \mathrm{E}+09$ & $4.3 \mathrm{E}+03$ \\
\hline $\mathrm{Rn}-222$ & $5.5 \mathrm{E}-04$ & $2.0 \mathrm{E}+12$ & $4.8 \mathrm{E}+06$ \\
\hline $\mathrm{Ru}-103$ & $1.1 \mathrm{E}-03$ & $4.1 \mathrm{E}+12$ & $9.5 \mathrm{E}+06$ \\
\hline Ru-94 & $4.2 \mathrm{E}-06$ & $1.6 \mathrm{E}+10$ & $3.6 \mathrm{E}+04$ \\
\hline Ru-97 & $4.1 \mathrm{E}-05$ & $1.5 \mathrm{E}+11$ & $3.5 \mathrm{E}+05$ \\
\hline Ru-105 & $1.0 \mathrm{E}-05$ & $3.7 \mathrm{E}+10$ & $8.7 E+04$ \\
\hline Ru-106 & $9.6 \mathrm{E}-03$ & $3.6 \mathrm{E}+13$ & $8.3 \mathrm{E}+07$ \\
\hline S-35 & $3.0 \mathrm{E}-03$ & $1.1 \mathrm{E}+13$ & $2.6 \mathrm{E}+07$ \\
\hline Sb-115 & $1.7 \mathrm{E}-06$ & $6.3 \mathrm{E}+09$ & $1.5 \mathrm{E}+04$ \\
\hline Sb-116 & $2.8 \mathrm{E}-06$ & $1.0 \mathrm{E}+10$ & $2.4 \mathrm{E}+04$ \\
\hline $\mathrm{Sb}-116 \mathrm{~m}$ & $8.5 \mathrm{E}-06$ & $3.1 \mathrm{E}+10$ & $7.4 \mathrm{E}+04$ \\
\hline Sb-117 & $1.1 \mathrm{E}-06$ & $4.1 E+09$ & $9.5 \mathrm{E}+03$ \\
\hline $\mathrm{Sb}-118 \mathrm{~m}$ & $2.2 \mathrm{E}-05$ & $8.1 E+10$ & $1.9 \mathrm{E}+05$ \\
\hline $\mathrm{Sb}-119$ & $5.6 \mathrm{E}-06$ & $2.1 \mathrm{E}+10$ & $4.8 \mathrm{E}+04$ \\
\hline
\end{tabular}


Table 1. Results of the Screening Analysis

\begin{tabular}{|c|c|c|c|}
\hline Radionuclide & $\begin{array}{c}\text { Level I } \\
\text { Screening Factor } \\
\left(\mathrm{Sv} / \mathrm{Bq}-\mathrm{m}^{-3}\right) \\
\end{array}$ & $\begin{array}{c}\text { Level I } \\
\text { Screening Factor } \\
\left(\mathrm{mrem} / \mathrm{Ci}-\mathrm{m}^{-3}\right) \\
\end{array}$ & $\begin{array}{l}\text { Screening } \\
\text { Dose } \\
\text { mrem/yr } \\
\end{array}$ \\
\hline Sb-120a & 5.9E-07 & $2.2 \mathrm{E}+09$ & $5.1 \mathrm{E}+03$ \\
\hline $\mathrm{Sb}-120 \mathrm{~b}$ & 7.2E-04 & $2.7 \mathrm{E}+12$ & $6.2 \mathrm{E}+06$ \\
\hline $\mathrm{Sb}-122$ & 2.3E-04 & $8.5 \mathrm{E}+11$ & $2.0 \mathrm{E}+06$ \\
\hline $\mathrm{Sb}-124$ & $5.3 \mathrm{E}-03$ & $2.0 \mathrm{E}+13$ & $4.6 \mathrm{E}+07$ \\
\hline $\mathrm{Sb}-124 \mathrm{~m}$ & $1.0 \mathrm{E}-07$ & $3.7 \mathrm{E}+08$ & $8.7 E+02$ \\
\hline Sb-124n & $1.6 \mathrm{E}-06$ & $5.9 \mathrm{E}+09$ & $1.4 \mathrm{E}+04$ \\
\hline $\mathrm{Sb}-125$ & $1.6 \mathrm{E}-02$ & $5.9 \mathrm{E}+13$ & $1.4 \mathrm{E}+08$ \\
\hline Sb-126 & $2.1 \mathrm{E}-03$ & $7.8 \mathrm{E}+12$ & $1.8 \mathrm{E}+07$ \\
\hline $\mathrm{Sb}-126 \mathrm{~m}$ & $2.6 \mathrm{E}-06$ & $9.6 \mathrm{E}+09$ & $2.2 \mathrm{E}+04$ \\
\hline $\mathrm{Sb}-127$ & $3.7 \mathrm{E}-04$ & $1.4 \mathrm{E}+12$ & $3.2 \mathrm{E}+06$ \\
\hline $\mathrm{Sb}-128 \mathrm{a}$ & $2.0 \mathrm{E}-06$ & $7.4 \mathrm{E}+09$ & $1.7 E+04$ \\
\hline $\mathrm{Sb}-128 \mathrm{~b}$ & $5.3 \mathrm{E}-05$ & $2.0 \mathrm{E}+11$ & $4.6 \mathrm{E}+05$ \\
\hline $\mathrm{Sb}-129$ & $1.5 \mathrm{E}-05$ & $5.6 \mathrm{E}+10$ & $1.3 \mathrm{E}+05$ \\
\hline Sb-130 & $7.1 \mathrm{E}-06$ & $2.6 \mathrm{E}+10$ & $6.1 \mathrm{E}+04$ \\
\hline Sb-131 & 5.9E-05 & $2.2 \mathrm{E}+11$ & $5.1 \mathrm{E}+05$ \\
\hline Sc-43 & $8.5 \mathrm{E}-06$ & $3.1 \mathrm{E}+10$ & $7.4 \mathrm{E}+04$ \\
\hline Sc-44 & $1.5 \mathrm{E}-05$ & $5.6 \mathrm{E}+10$ & $1.3 \mathrm{E}+05$ \\
\hline Sc- $44 m$ & $4.2 \mathrm{E}-04$ & $1.6 \mathrm{E}+12$ & $3.6 \mathrm{E}+06$ \\
\hline $\mathrm{Sc}-46$ & $6.6 \mathrm{E}-03$ & $2.4 \mathrm{E}+13$ & $5.7 \mathrm{E}+07$ \\
\hline Sc-47 & 8.8E-05 & $3.3 \mathrm{E}+11$ & $7.6 \mathrm{E}+05$ \\
\hline Sc-48 & $3.0 \mathrm{E}-04$ & $1.1 \mathrm{E}+12$ & $2.6 \mathrm{E}+06$ \\
\hline Sc-49 & $2.0 \mathrm{E}-07$ & $7.4 \mathrm{E}+08$ & $1.7 E+03$ \\
\hline $\mathrm{Se}-70$ & $7.1 \mathrm{E}-06$ & $2.6 \mathrm{E}+10$ & $6.1 \mathrm{E}+04$ \\
\hline $\mathrm{Se}-73$ & $1.6 \mathrm{E}-05$ & $5.9 E+10$ & $1.4 \mathrm{E}+05$ \\
\hline $\mathrm{Se}-73 \mathrm{~m}$ & $1.6 \mathrm{E}-06$ & $5.9 \mathrm{E}+09$ & $1.4 \mathrm{E}+04$ \\
\hline $\mathrm{Se}-75$ & $1.4 \mathrm{E}-02$ & $5.2 \mathrm{E}+13$ & $1.2 \mathrm{E}+08$ \\
\hline $\mathrm{Se}-77 \mathrm{~m}$ & $7.1 \mathrm{E}-10$ & $2.6 \mathrm{E}+06$ & $6.1 \mathrm{E}+00$ \\
\hline Se-79 & $1.7 \mathrm{E}-02$ & $6.3 E+13$ & $1.5 \mathrm{E}+08$ \\
\hline $\mathrm{Se}-81$ & 5.9E-08 & $2.2 \mathrm{E}+08$ & $5.1 \mathrm{E}+02$ \\
\hline $\mathrm{Se}-81 \mathrm{~m}$ & $2.6 \mathrm{E}-07$ & $9.6 \mathrm{E}+08$ & $2.2 \mathrm{E}+03$ \\
\hline $\mathrm{Se}-83$ & $3.9 \mathrm{E}-06$ & $1.4 \mathrm{E}+10$ & $3.4 \mathrm{E}+04$ \\
\hline Si-31 & 4.4E-07 & $1.6 \mathrm{E}+09$ & $3.8 \mathrm{E}+03$ \\
\hline Si-32 & $6.8 \mathrm{E}-02$ & $2.5 \mathrm{E}+14$ & $5.9 \mathrm{E}+08$ \\
\hline Sm-141 & $1.8 \mathrm{E}-06$ & $6.7 \mathrm{E}+09$ & $1.6 \mathrm{E}+04$ \\
\hline Sm-141m & $3.9 \mathrm{E}-06$ & $1.4 \mathrm{E}+10$ & $3.4 \mathrm{E}+04$ \\
\hline Sm-142 & $3.3 \mathrm{E}-06$ & $1.2 \mathrm{E}+10$ & $2.9 \mathrm{E}+04$ \\
\hline Sm-145 & $1.2 \mathrm{E}-03$ & $4.4 \mathrm{E}+12$ & $1.0 \mathrm{E}+07$ \\
\hline Sm-146 & $1.4 \mathrm{E}-01$ & $5.2 \mathrm{E}+14$ & $1.2 \mathrm{E}+09$ \\
\hline Sm-147 & $1.3 \mathrm{E}-01$ & $4.8 \mathrm{E}+14$ & $1.1 \mathrm{E}+09$ \\
\hline $\mathrm{Sm}-151$ & $1.6 \mathrm{E}-04$ & $5.9 \mathrm{E}+11$ & $1.4 \mathrm{E}+06$ \\
\hline Sm-153 & $5.6 \mathrm{E}-05$ & $2.1 \mathrm{E}+11$ & $4.8 \mathrm{E}+05$ \\
\hline Sm-155 & $2.5 \mathrm{E}-07$ & $9.3 \mathrm{E}+08$ & $2.2 \mathrm{E}+03$ \\
\hline Sm-156 & $4.5 \mathrm{E}-05$ & $1.7 \mathrm{E}+11$ & $3.9 \mathrm{E}+05$ \\
\hline Sn-110 & $1.3 \mathrm{E}-05$ & $4.8 \mathrm{E}+10$ & $1.1 \mathrm{E}+05$ \\
\hline Sn-111 & $1.7 \mathrm{E}-06$ & $6.3 \mathrm{E}+09$ & $1.5 \mathrm{E}+04$ \\
\hline Sn-113 & 2.3E-03 & $8.5 E+12$ & $2.0 \mathrm{E}+07$ \\
\hline
\end{tabular}


Table 1. Results of the Screening Analysis

\begin{tabular}{|c|c|c|c|}
\hline Radionuclide & $\begin{array}{c}\text { Level I } \\
\text { Screening Factor } \\
\left(\mathrm{Sv} / \mathrm{Bq}-\mathrm{m}^{-3}\right) \\
\end{array}$ & $\begin{array}{c}\text { Level I } \\
\text { Screening Factor } \\
\left(\mathrm{mrem} / \mathrm{Ci}-\mathrm{m}^{-3}\right) \\
\end{array}$ & $\begin{array}{l}\text { Screening } \\
\text { Dose } \\
\text { mrem/yr } \\
\end{array}$ \\
\hline Sn-117m & 6.1E-04 & $2.3 \mathrm{E}+12$ & $5.3 \mathrm{E}+06$ \\
\hline Sn-119m & $6.7 \mathrm{E}-04$ & $2.5 \mathrm{E}+12$ & $5.8 \mathrm{E}+06$ \\
\hline $\mathrm{Sn}-121$ & 8.9E-06 & $3.3 \mathrm{E}+10$ & $7.7 \mathrm{E}+04$ \\
\hline $\mathrm{Sn}-121 \mathrm{~m}$ & $3.7 \mathrm{E}-03$ & $1.4 \mathrm{E}+13$ & $3.2 \mathrm{E}+07$ \\
\hline $\mathrm{Sn}-123$ & $3.4 \mathrm{E}-03$ & $1.3 \mathrm{E}+13$ & $2.9 \mathrm{E}+07$ \\
\hline Sn-123m & 4.0E-07 & $1.5 \mathrm{E}+09$ & $3.5 \mathrm{E}+03$ \\
\hline $\mathrm{Sn}-125$ & $2.1 \mathrm{E}-03$ & $7.8 \mathrm{E}+12$ & $1.8 \mathrm{E}+07$ \\
\hline Sn-126 & $5.2 \mathrm{E}-01$ & $1.9 \mathrm{E}+15$ & $4.5 \mathrm{E}+09$ \\
\hline Sn-127 & $1.7 \mathrm{E}-05$ & $6.3 E+10$ & $1.5 \mathrm{E}+05$ \\
\hline Sn-128 & $6.2 \mathrm{E}-06$ & $2.3 \mathrm{E}+10$ & $5.4 \mathrm{E}+04$ \\
\hline Sr-80 & $5.7 E-06$ & $2.1 \mathrm{E}+10$ & $4.9 \mathrm{E}+04$ \\
\hline Sr-81 & 2.9E-06 & $1.1 \mathrm{E}+10$ & $2.5 \mathrm{E}+04$ \\
\hline Sr-82 & $8.4 \mathrm{E}-03$ & $3.1 \mathrm{E}+13$ & $7.3 \mathrm{E}+07$ \\
\hline Sr-83 & $1.8 \mathrm{E}-04$ & $6.7 \mathrm{E}+11$ & $1.6 \mathrm{E}+06$ \\
\hline $\mathrm{Sr}-85$ & $1.8 \mathrm{E}-03$ & $6.7 \mathrm{E}+12$ & $1.6 \mathrm{E}+07$ \\
\hline Sr-85m & $1.8 \mathrm{E}-06$ & $6.7 \mathrm{E}+09$ & $1.6 \mathrm{E}+04$ \\
\hline $\mathrm{Sr}-87 \mathrm{~m}$ & $1.9 \mathrm{E}-06$ & $7.0 \mathrm{E}+09$ & $1.6 \mathrm{E}+04$ \\
\hline Sr-89 & $3.6 \mathrm{E}-03$ & $1.3 \mathrm{E}+13$ & $3.1 \mathrm{E}+07$ \\
\hline Sr-90 & $1.9 \mathrm{E}-01$ & $7.0 \mathrm{E}+14$ & $1.6 \mathrm{E}+09$ \\
\hline Sr-91 & $3.2 \mathrm{E}-05$ & $1.2 \mathrm{E}+11$ & $2.8 \mathrm{E}+05$ \\
\hline Sr-92 & $9.0 \mathrm{E}-06$ & $3.3 \mathrm{E}+10$ & $7.8 \mathrm{E}+04$ \\
\hline Тa-172 & $5.0 \mathrm{E}-06$ & $1.9 \mathrm{E}+10$ & $4.3 \mathrm{E}+04$ \\
\hline Тa-173 & $8.7 E-06$ & $3.2 \mathrm{E}+10$ & $7.5 \mathrm{E}+04$ \\
\hline Тa-174 & $2.0 \mathrm{E}-06$ & $7.4 \mathrm{E}+09$ & $1.7 E+04$ \\
\hline Тa-175 & $2.5 \mathrm{E}-05$ & $9.3 \mathrm{E}+10$ & $2.2 \mathrm{E}+05$ \\
\hline Та-176 & $2.8 \mathrm{E}-05$ & $1.0 \mathrm{E}+11$ & $2.4 \mathrm{E}+05$ \\
\hline Ta-177 & $1.5 \mathrm{E}-05$ & $5.6 \mathrm{E}+10$ & $1.3 \mathrm{E}+05$ \\
\hline Ta-178a & 9.3E-08 & $3.4 \mathrm{E}+08$ & $8.0 \mathrm{E}+02$ \\
\hline Ta-178b & $5.0 \mathrm{E}-06$ & $1.9 \mathrm{E}+10$ & $4.3 \mathrm{E}+04$ \\
\hline Та-179 & $9.2 \mathrm{E}-04$ & $3.4 \mathrm{E}+12$ & $8.0 \mathrm{E}+06$ \\
\hline Ta-180 & $1.5 \mathrm{E}-01$ & $5.6 \mathrm{E}+14$ & $1.3 \mathrm{E}+09$ \\
\hline Ta-180m & $1.0 \mathrm{E}-06$ & $3.7 \mathrm{E}+09$ & $8.7 \mathrm{E}+03$ \\
\hline Ta-182 & $6.0 \mathrm{E}-03$ & $2.2 \mathrm{E}+13$ & $5.2 \mathrm{E}+07$ \\
\hline Ta-182m & $9.2 \mathrm{E}-07$ & $3.4 \mathrm{E}+09$ & $8.0 \mathrm{E}+03$ \\
\hline Тa-183 & $3.0 \mathrm{E}-04$ & $1.1 \mathrm{E}+12$ & $2.6 \mathrm{E}+06$ \\
\hline Тa-184 & $2.8 \mathrm{E}-05$ & $1.0 \mathrm{E}+11$ & $2.4 \mathrm{E}+05$ \\
\hline Та-185 & $1.3 \mathrm{E}-06$ & $4.8 \mathrm{E}+09$ & $1.1 \mathrm{E}+04$ \\
\hline Та-186 & $1.6 \mathrm{E}-06$ & $5.9 \mathrm{E}+09$ & $1.4 \mathrm{E}+04$ \\
\hline Tb-147 & $1.2 \mathrm{E}-05$ & $4.4 \mathrm{E}+10$ & $1.0 \mathrm{E}+05$ \\
\hline Tb-149 & $3.4 \mathrm{E}-05$ & $1.3 \mathrm{E}+11$ & $2.9 \mathrm{E}+05$ \\
\hline Tb-150 & $1.1 \mathrm{E}-05$ & $4.1 \mathrm{E}+10$ & $9.5 \mathrm{E}+04$ \\
\hline Tb-151 & $3.3 \mathrm{E}-05$ & $1.2 \mathrm{E}+11$ & $2.9 \mathrm{E}+05$ \\
\hline Tb-153 & 5.3E-05 & $2.0 \mathrm{E}+11$ & $4.6 \mathrm{E}+05$ \\
\hline Tb-154 & $8.5 \mathrm{E}-05$ & $3.1 \mathrm{E}+11$ & $7.4 \mathrm{E}+05$ \\
\hline Tb-155 & $7.0 \mathrm{E}-05$ & $2.6 \mathrm{E}+11$ & $6.1 \mathrm{E}+05$ \\
\hline Tb-156 & $5.5 \mathrm{E}-04$ & $2.0 \mathrm{E}+12$ & $4.8 \mathrm{E}+06$ \\
\hline
\end{tabular}


Table 1. Results of the Screening Analysis

\begin{tabular}{|c|c|c|c|}
\hline Radionuclide & $\begin{array}{c}\text { Level I } \\
\text { Screening Factor } \\
\left(\mathrm{Sv} / \mathrm{Bq}-\mathrm{m}^{-3}\right) \\
\end{array}$ & $\begin{array}{c}\text { Level I } \\
\text { Screening Factor } \\
\left(\mathrm{mrem} / \mathrm{Ci}-\mathrm{m}^{-3}\right) \\
\end{array}$ & $\begin{array}{l}\text { Screening } \\
\text { Dose } \\
\text { mrem/yr } \\
\end{array}$ \\
\hline Tb-156m & $1.1 \mathrm{E}-04$ & $4.1 \mathrm{E}+11$ & $9.5 \mathrm{E}+05$ \\
\hline Tb-156n & $2.2 \mathrm{E}-05$ & $8.1 \mathrm{E}+10$ & $1.9 \mathrm{E}+05$ \\
\hline Tb-157 & $1.1 \mathrm{E}-03$ & $4.1 \mathrm{E}+12$ & $9.5 \mathrm{E}+06$ \\
\hline Tb-158 & $1.8 \mathrm{E}-01$ & $6.7 \mathrm{E}+14$ & $1.6 \mathrm{E}+09$ \\
\hline Tb-160 & 3.9E-03 & $1.4 \mathrm{E}+13$ & $3.4 \mathrm{E}+07$ \\
\hline Tb-161 & $2.0 \mathrm{E}-04$ & $7.4 \mathrm{E}+11$ & $1.7 E+06$ \\
\hline Тc-93 & 7.3E-06 & $2.7 \mathrm{E}+10$ & $6.3 \mathrm{E}+04$ \\
\hline Tc-93m & $2.9 \mathrm{E}-06$ & $1.1 \mathrm{E}+10$ & $2.5 \mathrm{E}+04$ \\
\hline Tc-94 & $2.4 \mathrm{E}-05$ & $8.9 \mathrm{E}+10$ & $2.1 \mathrm{E}+05$ \\
\hline Tc-94m & 4.9E-06 & $1.8 \mathrm{E}+10$ & $4.2 \mathrm{E}+04$ \\
\hline Tc-95 & $2.8 \mathrm{E}-05$ & $1.0 \mathrm{E}+11$ & $2.4 \mathrm{E}+05$ \\
\hline Tc-95m & $2.1 \mathrm{E}-03$ & $7.8 \mathrm{E}+12$ & $1.8 \mathrm{E}+07$ \\
\hline Тc-96 & $5.4 \mathrm{E}-04$ & $2.0 \mathrm{E}+12$ & $4.7 \mathrm{E}+06$ \\
\hline Тc-96m & $4.5 \mathrm{E}-06$ & $1.7 \mathrm{E}+10$ & $3.9 \mathrm{E}+04$ \\
\hline Tc-97 & $5.2 \mathrm{E}-03$ & $1.9 \mathrm{E}+13$ & $4.5 \mathrm{E}+07$ \\
\hline Tc-97m & $7.1 \mathrm{E}-04$ & $2.6 \mathrm{E}+12$ & $6.1 \mathrm{E}+06$ \\
\hline Tc-98 & $4.4 \mathrm{E}-01$ & $1.6 \mathrm{E}+15$ & $3.8 \mathrm{E}+09$ \\
\hline Tc-99 & $3.4 \mathrm{E}-02$ & $1.3 \mathrm{E}+14$ & $2.9 \mathrm{E}+08$ \\
\hline Tc-99m & $1.6 \mathrm{E}-06$ & $5.9 \mathrm{E}+09$ & $1.4 \mathrm{E}+04$ \\
\hline Tc-101 & $4.5 \mathrm{E}-07$ & $1.7 \mathrm{E}+09$ & $3.9 \mathrm{E}+03$ \\
\hline Tc-104 & $3.0 \mathrm{E}-06$ & $1.1 \mathrm{E}+10$ & $2.6 \mathrm{E}+04$ \\
\hline Te-116 & $9.4 \mathrm{E}-06$ & $3.5 E+10$ & $8.1 \mathrm{E}+04$ \\
\hline Тe-121 & $5.6 \mathrm{E}-04$ & $2.1 \mathrm{E}+12$ & $4.8 \mathrm{E}+06$ \\
\hline $\mathrm{Te}-121 \mathrm{~m}$ & $5.5 \mathrm{E}-03$ & $2.0 \mathrm{E}+13$ & $4.8 \mathrm{E}+07$ \\
\hline $\mathrm{Te}-123$ & $3.2 \mathrm{E}-03$ & $1.2 \mathrm{E}+13$ & $2.8 \mathrm{E}+07$ \\
\hline $\mathrm{Te}-123 \mathrm{~m}$ & $1.6 \mathrm{E}-03$ & $5.9 \mathrm{E}+12$ & $1.4 \mathrm{E}+07$ \\
\hline Te- $125 \mathrm{~m}$ & $6.9 \mathrm{E}-04$ & $2.6 \mathrm{E}+12$ & $6.0 \mathrm{E}+06$ \\
\hline Te-127 & $1.2 \mathrm{E}-06$ & $4.4 \mathrm{E}+09$ & $1.0 \mathrm{E}+04$ \\
\hline $\mathrm{Te}-127 \mathrm{~m}$ & $1.9 \mathrm{E}-03$ & $7.0 \mathrm{E}+12$ & $1.6 \mathrm{E}+07$ \\
\hline Te-129 & $3.4 \mathrm{E}-07$ & $1.3 \mathrm{E}+09$ & $2.9 \mathrm{E}+03$ \\
\hline Te-129m & $2.3 \mathrm{E}-03$ & $8.5 \mathrm{E}+12$ & $2.0 \mathrm{E}+07$ \\
\hline $\mathrm{Te}-131$ & $6.3 \mathrm{E}-05$ & $2.3 E+11$ & $5.5 \mathrm{E}+05$ \\
\hline Тe-131m & $4.2 \mathrm{E}-03$ & $1.6 \mathrm{E}+13$ & $3.6 \mathrm{E}+07$ \\
\hline Te-132 & $7.2 \mathrm{E}-04$ & $2.7 \mathrm{E}+12$ & $6.2 \mathrm{E}+06$ \\
\hline Te-133 & $3.2 \mathrm{E}-06$ & $1.2 \mathrm{E}+10$ & $2.8 \mathrm{E}+04$ \\
\hline Te-133m & $1.6 \mathrm{E}-05$ & $5.9 \mathrm{E}+10$ & $1.4 \mathrm{E}+05$ \\
\hline Te-134 & $5.2 \mathrm{E}-06$ & $1.9 \mathrm{E}+10$ & $4.5 \mathrm{E}+04$ \\
\hline Th-226 & $6.5 \mathrm{E}-05$ & $2.4 \mathrm{E}+11$ & $5.6 \mathrm{E}+05$ \\
\hline Th-227 & $6.4 \mathrm{E}-02$ & $2.4 \mathrm{E}+14$ & $5.5 \mathrm{E}+08$ \\
\hline Th-228 & 8.0E-01 & $3.0 \mathrm{E}+15$ & $6.9 \mathrm{E}+09$ \\
\hline Th-229 & $3.4 \mathrm{E}+00$ & $1.3 \mathrm{E}+16$ & $2.9 \mathrm{E}+10$ \\
\hline Th-230 & $4.9 \mathrm{E}-01$ & $1.8 \mathrm{E}+15$ & $4.2 \mathrm{E}+09$ \\
\hline Th-231 & $1.1 \mathrm{E}-05$ & $4.1 \mathrm{E}+10$ & $9.5 \mathrm{E}+04$ \\
\hline Th-232 & $2.6 \mathrm{E}+00$ & $9.6 \mathrm{E}+15$ & $2.2 \mathrm{E}+10$ \\
\hline Th-234 & $1.8 \mathrm{E}-03$ & $6.7 \mathrm{E}+12$ & $1.6 \mathrm{E}+07$ \\
\hline Ti-44 & 4.5E-01 & $1.7 \mathrm{E}+15$ & $3.9 \mathrm{E}+09$ \\
\hline
\end{tabular}


Table 1. Results of the Screening Analysis

\begin{tabular}{|c|c|c|c|}
\hline Radionuclide & $\begin{array}{c}\text { Level I } \\
\text { Screening Factor } \\
\left(\mathrm{Sv} / \mathrm{Bq}-\mathrm{m}^{-3}\right) \\
\end{array}$ & $\begin{array}{c}\text { Level I } \\
\text { Screening Factor } \\
\left(\mathrm{mrem} / \mathrm{Ci}-\mathrm{m}^{-3}\right) \\
\end{array}$ & $\begin{array}{l}\text { Screening } \\
\text { Dose } \\
\text { mrem/yr } \\
\end{array}$ \\
\hline Ti-45 & 5.5E-06 & $2.0 \mathrm{E}+10$ & $4.8 \mathrm{E}+04$ \\
\hline Tl-194 & $1.6 \mathrm{E}-06$ & $5.9 \mathrm{E}+09$ & $1.4 \mathrm{E}+04$ \\
\hline $\mathrm{Tl}-194 \mathrm{~m}$ & $4.6 \mathrm{E}-06$ & $1.7 \mathrm{E}+10$ & $4.0 \mathrm{E}+04$ \\
\hline Tl-195 & $4.4 \mathrm{E}-06$ & $1.6 \mathrm{E}+10$ & $3.8 \mathrm{E}+04$ \\
\hline Tl-197 & $4.0 \mathrm{E}-06$ & $1.5 \mathrm{E}+10$ & $3.5 \mathrm{E}+04$ \\
\hline Tl-198 & $1.8 \mathrm{E}-05$ & $6.7 \mathrm{E}+10$ & $1.6 \mathrm{E}+05$ \\
\hline $\mathrm{Tl}-198 \mathrm{~m}$ & $7.7 \mathrm{E}-06$ & $2.9 \mathrm{E}+10$ & $6.7 E+04$ \\
\hline Tl-199 & $3.5 \mathrm{E}-06$ & $1.3 \mathrm{E}+10$ & $3.0 \mathrm{E}+04$ \\
\hline Tl-200 & $5.8 \mathrm{E}-05$ & $2.1 \mathrm{E}+11$ & $5.0 \mathrm{E}+05$ \\
\hline Tl-201 & $2.6 \mathrm{E}-05$ & $9.6 \mathrm{E}+10$ & $2.2 \mathrm{E}+05$ \\
\hline Tl-202 & $5.1 \mathrm{E}-04$ & $1.9 \mathrm{E}+12$ & $4.4 \mathrm{E}+06$ \\
\hline Tl-204 & $1.9 \mathrm{E}-03$ & $7.0 \mathrm{E}+12$ & $1.6 \mathrm{E}+07$ \\
\hline Tl-206 & $4.6 \mathrm{E}-11$ & $1.7 \mathrm{E}+05$ & 4.0E-01 \\
\hline Tl-207 & 1.1E-09 & $4.1 \mathrm{E}+06$ & $9.5 \mathrm{E}+00$ \\
\hline Tl-208 & $9.9 \mathrm{E}-07$ & $3.7 \mathrm{E}+09$ & $8.6 \mathrm{E}+03$ \\
\hline Tl-209 & $3.1 \mathrm{E}-07$ & $1.1 \mathrm{E}+09$ & $2.7 \mathrm{E}+03$ \\
\hline Tm-162 & $2.8 \mathrm{E}-06$ & $1.0 \mathrm{E}+10$ & $2.4 \mathrm{E}+04$ \\
\hline Tm-166 & $2.4 \mathrm{E}-05$ & $8.9 \mathrm{E}+10$ & $2.1 \mathrm{E}+05$ \\
\hline Tm-167 & $2.4 \mathrm{E}-04$ & $8.9 E+11$ & $2.1 \mathrm{E}+06$ \\
\hline Tm-170 & $1.2 \mathrm{E}-03$ & $4.4 \mathrm{E}+12$ & $1.0 \mathrm{E}+07$ \\
\hline Tm-171 & $1.3 \mathrm{E}-04$ & $4.8 \mathrm{E}+11$ & $1.1 \mathrm{E}+06$ \\
\hline Tm-172 & $2.1 \mathrm{E}-04$ & $7.8 \mathrm{E}+11$ & $1.8 \mathrm{E}+06$ \\
\hline Tm-173 & $7.1 \mathrm{E}-06$ & $2.6 \mathrm{E}+10$ & $6.1 \mathrm{E}+04$ \\
\hline Tm-175 & $1.6 \mathrm{E}-06$ & $5.9 \mathrm{E}+09$ & $1.4 \mathrm{E}+04$ \\
\hline $\mathrm{U}-230$ & 8.3E-02 & $3.1 \mathrm{E}+14$ & 7.2E+08 \\
\hline U-231 & $6.4 \mathrm{E}-05$ & $2.4 \mathrm{E}+11$ & $5.5 \mathrm{E}+05$ \\
\hline U-232 & $1.9 \mathrm{E}+00$ & $7.0 \mathrm{E}+15$ & $1.6 \mathrm{E}+10$ \\
\hline $\mathrm{U}-233$ & $3.2 \mathrm{E}-01$ & $1.2 \mathrm{E}+15$ & $2.8 \mathrm{E}+09$ \\
\hline U-234 & $3.1 \mathrm{E}-01$ & $1.1 \mathrm{E}+15$ & $2.7 \mathrm{E}+09$ \\
\hline U-235 & $3.4 \mathrm{E}-01$ & $1.3 \mathrm{E}+15$ & $2.9 \mathrm{E}+09$ \\
\hline U-236 & $3.0 \mathrm{E}-01$ & $1.1 \mathrm{E}+15$ & $2.6 \mathrm{E}+09$ \\
\hline U-237 & $2.5 \mathrm{E}-04$ & $9.3 \mathrm{E}+11$ & $2.2 \mathrm{E}+06$ \\
\hline U-238 & 2.9E-01 & $1.1 \mathrm{E}+15$ & $2.5 \mathrm{E}+09$ \\
\hline U-239 & $7.0 \mathrm{E}-07$ & $2.6 \mathrm{E}+09$ & $6.1 \mathrm{E}+03$ \\
\hline $\mathrm{U}-240$ & $2.1 \mathrm{E}-05$ & $7.8 \mathrm{E}+10$ & $1.8 \mathrm{E}+05$ \\
\hline V-47 & $2.0 \mathrm{E}-06$ & $7.4 \mathrm{E}+09$ & $1.7 \mathrm{E}+04$ \\
\hline$V-48$ & $2.8 \mathrm{E}-03$ & $1.0 \mathrm{E}+13$ & $2.4 \mathrm{E}+07$ \\
\hline$V-49$ & $2.3 \mathrm{E}-05$ & $8.5 \mathrm{E}+10$ & $2.0 \mathrm{E}+05$ \\
\hline W-176 & $8.1 \mathrm{E}-06$ & $3.0 \mathrm{E}+10$ & $7.0 \mathrm{E}+04$ \\
\hline W-177 & $4.9 \mathrm{E}-06$ & $1.8 \mathrm{E}+10$ & $4.2 \mathrm{E}+04$ \\
\hline W-178 & $5.3 \mathrm{E}-04$ & $2.0 \mathrm{E}+12$ & $4.6 \mathrm{E}+06$ \\
\hline W-179 & $1.5 \mathrm{E}-07$ & $5.6 \mathrm{E}+08$ & $1.3 \mathrm{E}+03$ \\
\hline W-181 & $4.4 \mathrm{E}-04$ & $1.6 \mathrm{E}+12$ & $3.8 \mathrm{E}+06$ \\
\hline W-185 & $1.5 \mathrm{E}-03$ & $5.6 \mathrm{E}+12$ & $1.3 \mathrm{E}+07$ \\
\hline W-187 & $3.6 \mathrm{E}-05$ & $1.3 \mathrm{E}+11$ & $3.1 \mathrm{E}+05$ \\
\hline W-188 & 8.0E-03 & $3.0 \mathrm{E}+13$ & $6.9 \mathrm{E}+07$ \\
\hline
\end{tabular}


Table 1. Results of the Screening Analysis

\begin{tabular}{|c|c|c|c|}
\hline Radionuclide & $\begin{array}{c}\text { Level I } \\
\text { Screening Factor } \\
\left(\mathrm{Sv} / \mathrm{Bq}-\mathrm{m}^{-3}\right) \\
\end{array}$ & $\begin{array}{c}\text { Level I } \\
\text { Screening Factor } \\
\left(\mathrm{mrem} / \mathrm{Ci}-\mathrm{m}^{-3}\right) \\
\end{array}$ & $\begin{array}{l}\text { Screening } \\
\text { Dose } \\
\text { mrem/yr } \\
\end{array}$ \\
\hline Xe-120 & 3.4E-06 & $1.3 \mathrm{E}+10$ & $2.9 \mathrm{E}+04$ \\
\hline $\mathrm{Xe}-121$ & $3.5 \mathrm{E}-06$ & $1.3 \mathrm{E}+10$ & $3.0 \mathrm{E}+04$ \\
\hline $\mathrm{Xe}-122$ & $3.1 \mathrm{E}-05$ & $1.1 \mathrm{E}+11$ & $2.7 \mathrm{E}+05$ \\
\hline $\mathrm{Xe}-123$ & $1.8 \mathrm{E}-06$ & $6.7 \mathrm{E}+09$ & $1.6 \mathrm{E}+04$ \\
\hline $\mathrm{Xe}-125$ & $5.8 \mathrm{E}-04$ & $2.1 \mathrm{E}+12$ & $5.0 \mathrm{E}+06$ \\
\hline $\mathrm{Xe}-127$ & $3.3 \mathrm{E}-07$ & $1.2 \mathrm{E}+09$ & $2.9 \mathrm{E}+03$ \\
\hline $\mathrm{Xe}-129 \mathrm{~m}$ & 2.9E-08 & $1.1 \mathrm{E}+08$ & $2.5 \mathrm{E}+02$ \\
\hline $\mathrm{Xe}-131 \mathrm{~m}$ & $1.1 \mathrm{E}-08$ & $4.1 \mathrm{E}+07$ & $9.5 \mathrm{E}+01$ \\
\hline $\mathrm{Xe}-133$ & 4.3E-08 & $1.6 \mathrm{E}+08$ & $3.7 \mathrm{E}+02$ \\
\hline $\mathrm{Xe}-133 \mathrm{~m}$ & $3.7 \mathrm{E}-08$ & $1.4 \mathrm{E}+08$ & $3.2 \mathrm{E}+02$ \\
\hline $\mathrm{Xe}-135$ & $3.0 \mathrm{E}-07$ & $1.1 \mathrm{E}+09$ & $2.6 \mathrm{E}+03$ \\
\hline $\mathrm{Xe}-135 \mathrm{~m}$ & $3.7 \mathrm{E}-07$ & $1.4 \mathrm{E}+09$ & $3.2 \mathrm{E}+03$ \\
\hline Xe-138 & $2.2 \mathrm{E}-06$ & $8.1 \mathrm{E}+09$ & $1.9 \mathrm{E}+04$ \\
\hline Y-86 & 8.7E-05 & $3.2 \mathrm{E}+11$ & $7.5 \mathrm{E}+05$ \\
\hline Y-86m & $5.1 \mathrm{E}-06$ & $1.9 \mathrm{E}+10$ & $4.4 \mathrm{E}+04$ \\
\hline Y-87 & $1.6 \mathrm{E}-04$ & $5.9 \mathrm{E}+11$ & $1.4 \mathrm{E}+06$ \\
\hline Y-88 & $1.0 \mathrm{E}-02$ & $3.7 E+13$ & $8.7 E+07$ \\
\hline Y-90 & $2.8 \mathrm{E}-04$ & $1.0 \mathrm{E}+12$ & $2.4 \mathrm{E}+06$ \\
\hline Y-90m & $1.8 \mathrm{E}-05$ & $6.7 \mathrm{E}+10$ & $1.6 \mathrm{E}+05$ \\
\hline Y-91 & $1.8 \mathrm{E}-03$ & $6.7 \mathrm{E}+12$ & $1.6 \mathrm{E}+07$ \\
\hline $\mathrm{Y}-91 \mathrm{~m}$ & $2.4 \mathrm{E}-06$ & $8.9 \mathrm{E}+09$ & $2.1 \mathrm{E}+04$ \\
\hline Y-92 & $3.1 \mathrm{E}-06$ & $1.1 \mathrm{E}+10$ & $2.7 \mathrm{E}+04$ \\
\hline Y-93 & $9.5 \mathrm{E}-06$ & $3.5 \mathrm{E}+10$ & $8.2 \mathrm{E}+04$ \\
\hline Y-94 & $1.7 \mathrm{E}-06$ & $6.3 \mathrm{E}+09$ & $1.5 \mathrm{E}+04$ \\
\hline Y-95 & $1.5 \mathrm{E}-06$ & $5.6 \mathrm{E}+09$ & $1.3 \mathrm{E}+04$ \\
\hline Yb-162 & $1.5 \mathrm{E}-06$ & $5.6 \mathrm{E}+09$ & $1.3 \mathrm{E}+04$ \\
\hline Yb-166 & $2.4 \mathrm{E}-04$ & $8.9 \mathrm{E}+11$ & $2.1 \mathrm{E}+06$ \\
\hline Yb-167 & $6.8 \mathrm{E}-07$ & $2.5 \mathrm{E}+09$ & $5.9 \mathrm{E}+03$ \\
\hline Yb-169 & 8.3E-04 & $3.1 \mathrm{E}+12$ & $7.2 \mathrm{E}+06$ \\
\hline Yb-175 & $8.1 \mathrm{E}-05$ & $3.0 \mathrm{E}+11$ & $7.0 \mathrm{E}+05$ \\
\hline Yb-177 & $2.7 \mathrm{E}-06$ & $1.0 \mathrm{E}+10$ & $2.3 \mathrm{E}+04$ \\
\hline Yb-178 & 7.1E-07 & $2.6 \mathrm{E}+09$ & $6.1 \mathrm{E}+03$ \\
\hline Zn-62 & $3.0 \mathrm{E}-05$ & $1.1 \mathrm{E}+11$ & $2.6 \mathrm{E}+05$ \\
\hline $\mathrm{Zn}-63$ & $2.4 \mathrm{E}-06$ & $8.9 \mathrm{E}+09$ & $2.1 \mathrm{E}+04$ \\
\hline $\mathrm{Zn}-65$ & 2.7E-02 & $1.0 \mathrm{E}+14$ & $2.3 \mathrm{E}+08$ \\
\hline Zn-69 & 7.7E-08 & $2.9 \mathrm{E}+08$ & $6.7 E+02$ \\
\hline $\mathrm{Zn}-69 \mathrm{~m}$ & $1.6 \mathrm{E}-05$ & $5.9 \mathrm{E}+10$ & $1.4 \mathrm{E}+05$ \\
\hline $\mathrm{Zn}-71 \mathrm{~m}$ & $1.2 \mathrm{E}-05$ & $4.4 \mathrm{E}+10$ & $1.0 \mathrm{E}+05$ \\
\hline $\mathrm{Zn}-72$ & $6.1 \mathrm{E}-04$ & $2.3 \mathrm{E}+12$ & $5.3 \mathrm{E}+06$ \\
\hline Zr-86 & $1.1 \mathrm{E}-04$ & $4.1 \mathrm{E}+11$ & $9.5 \mathrm{E}+05$ \\
\hline Zr-88 & 8.7E-03 & $3.2 \mathrm{E}+13$ & $7.5 \mathrm{E}+07$ \\
\hline Zr-89 & 2.2E-04 & $8.1 \mathrm{E}+11$ & $1.9 \mathrm{E}+06$ \\
\hline Zr-93 & $7.4 \mathrm{E}-04$ & $2.7 \mathrm{E}+12$ & $6.4 \mathrm{E}+06$ \\
\hline Zr-95 & 4.1E-03 & $1.5 \mathrm{E}+13$ & $3.5 \mathrm{E}+07$ \\
\hline Zr-97 & 7.2E-05 & $2.7 \mathrm{E}+11$ & $6.2 \mathrm{E}+05$ \\
\hline
\end{tabular}


Table 2. Trigger Values

\begin{tabular}{|c|c|}
\hline Radionuclide & $\begin{array}{c}\text { Trigger } \\
\text { Value } \\
\mathrm{Ci}\end{array}$ \\
\hline Ar-37 & $6.8 \mathrm{E}+07$ \\
\hline Ar- 41 & $7.7 \mathrm{E}+01$ \\
\hline As-69 & $6.4 \mathrm{E}+01$ \\
\hline As-70 & $1.2 \mathrm{E}+01$ \\
\hline As-71 & $1.1 \mathrm{E}+00$ \\
\hline As-72 & 9.6E-01 \\
\hline As-73 & $3.7 \mathrm{E}-01$ \\
\hline As-74 & 8.3E-02 \\
\hline As-76 & $1.9 \mathrm{E}+00$ \\
\hline As-77 & $5.5 \mathrm{E}+00$ \\
\hline As-78 & $2.4 \mathrm{E}+01$ \\
\hline At-207 & $7.2 \mathrm{E}+00$ \\
\hline At-211 & $4.8 \mathrm{E}-01$ \\
\hline At-215 & $2.0 \mathrm{E}+10$ \\
\hline At-216 & $3.7 \mathrm{E}+07$ \\
\hline At-217 & $3.6 \mathrm{E}+05$ \\
\hline At-218 & $5.3 \mathrm{E}+03$ \\
\hline Br-74 & $1.5 \mathrm{E}+01$ \\
\hline $\mathrm{Br}-74 \mathrm{~m}$ & $1.3 \mathrm{E}+01$ \\
\hline $\mathrm{Br}-75$ & $9.6 \mathrm{E}+00$ \\
\hline $\mathrm{Br}-76$ & $1.4 \mathrm{E}+00$ \\
\hline Br-77 & $1.8 \mathrm{E}+00$ \\
\hline $\mathrm{Br}-80$ & $7.7 \mathrm{E}+02$ \\
\hline $\mathrm{Br}-80 \mathrm{~m}$ & $7.2 \mathrm{E}+01$ \\
\hline $\mathrm{Br}-82$ & $5.0 \mathrm{E}-01$ \\
\hline $\mathrm{Br}-83$ & $4.8 \mathrm{E}+02$ \\
\hline $\mathrm{Br}-84$ & $3.2 \mathrm{E}+01$ \\
\hline C-11 & $7.7 \mathrm{E}+01$ \\
\hline $\mathrm{Cl}-38$ & $3.5 \mathrm{E}+01$ \\
\hline $\mathrm{Cl}-39$ & $3.0 \mathrm{E}+01$ \\
\hline F-18 & $2.8 \mathrm{E}+01$ \\
\hline Ge-66 & $9.6 \mathrm{E}+00$ \\
\hline Ge-67 & $5.3 \mathrm{E}+01$ \\
\hline Ge- 68 & $8.9 \mathrm{E}-03$ \\
\hline Ge-69 & $1.6 \mathrm{E}+00$ \\
\hline Ge-71 & $1.1 \mathrm{E}+01$ \\
\hline Ge-75 & $4.3 \mathrm{E}+02$ \\
\hline $\mathrm{Ge}-77$ & $4.1 \mathrm{E}+00$ \\
\hline Ge-78 & $2.7 \mathrm{E}+01$ \\
\hline Hg-193 & $3.7 E+01$ \\
\hline $\mathrm{Hg}-193 \mathrm{~m}$ & $4.3 \mathrm{E}+00$ \\
\hline Hg-194 & $2.5 \mathrm{E}-04$ \\
\hline Hg-195 & $1.9 \mathrm{E}+01$ \\
\hline $\mathrm{Hg}-195 \mathrm{~m}$ & $1.7 \mathrm{E}+00$ \\
\hline $\mathrm{Hg}-197$ & $3.1 \mathrm{E}+00$ \\
\hline $\mathrm{Hg}-197 \mathrm{~m}$ & $3.9 \mathrm{E}+00$ \\
\hline
\end{tabular}

Table 2. Trigger Values

\begin{tabular}{|c|c|}
\hline Radionuclide & $\begin{array}{c}\text { Trigger } \\
\text { Value } \\
\mathrm{Ci} \\
\end{array}$ \\
\hline Hg-199m & $2.1 \mathrm{E}+02$ \\
\hline $\mathrm{Hg}-203$ & $6.4 \mathrm{E}-02$ \\
\hline I-120 & $1.2 \mathrm{E}+01$ \\
\hline $\mathrm{I}-120 \mathrm{~m}$ & $8.3 \mathrm{E}+00$ \\
\hline $\mathrm{I}-121$ & $2.3 \mathrm{E}+01$ \\
\hline I-122 & $3.5 E+02$ \\
\hline I-123 & $1.5 \mathrm{E}+01$ \\
\hline I-124 & $1.4 \mathrm{E}-02$ \\
\hline I-125 & 2.2E-03 \\
\hline I-126 & $2.1 \mathrm{E}-03$ \\
\hline I-128 & $5.3 \mathrm{E}+02$ \\
\hline I-130 & $1.6 \mathrm{E}+00$ \\
\hline I-131 & 4.1E-03 \\
\hline I-132 & $9.6 \mathrm{E}+00$ \\
\hline $\mathrm{I}-132 \mathrm{~m}$ & $1.9 \mathrm{E}+01$ \\
\hline I-133 & 5.3E-01 \\
\hline I-134 & $1.8 \mathrm{E}+01$ \\
\hline $\mathrm{I}-135$ & $4.6 \mathrm{E}+00$ \\
\hline $\mathrm{Kr}-74$ & $3.6 \mathrm{E}+01$ \\
\hline $\mathrm{Kr}-76$ & $1.7 \mathrm{E}+00$ \\
\hline $\mathrm{Kr}-77$ & $4.3 \mathrm{E}+01$ \\
\hline Kr-79 & $3.7 \mathrm{E}+02$ \\
\hline $\mathrm{Kr}-81$ & $8.9 \mathrm{E}+03$ \\
\hline $\mathrm{Kr}-81 \mathrm{~m}$ & $2.6 \mathrm{E}+14$ \\
\hline $\mathrm{Kr}-83 \mathrm{~m}$ & $1.1 \mathrm{E}+06$ \\
\hline $\mathrm{Kr}-85$ & $4.1 \mathrm{E}+04$ \\
\hline $\mathrm{Kr}-85 \mathrm{~m}$ & $5.8 \mathrm{E}+02$ \\
\hline $\mathrm{Kr}-87$ & $1.2 \mathrm{E}+02$ \\
\hline $\mathrm{Kr}-88$ & $2.1 \mathrm{E}+01$ \\
\hline $\mathrm{N}-13$ & $1.2 \mathrm{E}+02$ \\
\hline O-15 & $8.9 \mathrm{E}+02$ \\
\hline P-30 & $6.1 E+02$ \\
\hline P-32 & $1.6 \mathrm{E}-02$ \\
\hline P-33 & $1.2 \mathrm{E}-01$ \\
\hline S-35 & $3.9 \mathrm{E}-02$ \\
\hline Sb-115 & $6.8 \mathrm{E}+01$ \\
\hline $\mathrm{Sb}-116$ & $4.1 \mathrm{E}+01$ \\
\hline $\mathrm{Sb}-116 \mathrm{~m}$ & $1.4 \mathrm{E}+01$ \\
\hline Sb-117 & $1.1 \mathrm{E}+02$ \\
\hline $\mathrm{Sb}-118 \mathrm{~m}$ & $5.3 \mathrm{E}+00$ \\
\hline $\mathrm{Sb}-119$ & $2.1 \mathrm{E}+01$ \\
\hline Sb-120a & $2.0 \mathrm{E}+02$ \\
\hline Sb-120b & $1.6 \mathrm{E}-01$ \\
\hline $\mathrm{Sb}-122$ & $5.0 \mathrm{E}-01$ \\
\hline Sb-124 & $2.2 \mathrm{E}-02$ \\
\hline Sb-124m & $1.2 \mathrm{E}+03$ \\
\hline
\end{tabular}


Table 2. Trigger Values

\begin{tabular}{lc}
\hline \hline & $\begin{array}{c}\text { Trigger } \\
\text { Value }\end{array}$ \\
Radionuclide & Ci \\
\hline \hline Sb-124n & $7.2 \mathrm{E}+01$ \\
Sb-127 & $3.1 \mathrm{E}-01$ \\
Sb-128a & $5.8 \mathrm{E}+01$ \\
Sb-128b & $2.2 \mathrm{E}+00$ \\
Sb-129 & $7.7 \mathrm{E}+00$ \\
Sb-130 & $1.6 \mathrm{E}+01$ \\
Sb-131 & $2.0 \mathrm{E}+00$ \\
Se-70 & $1.6 \mathrm{E}+01$ \\
Se-73 & $7.2 \mathrm{E}+00$ \\
Se-73m & $7.2 \mathrm{E}+01$ \\
Se-75 & $8.3 \mathrm{E}-03$ \\
Se-77m & $1.6 \mathrm{E}+05$ \\
Se- 81 & $2.0 \mathrm{E}+03$ \\
Se- $81 \mathrm{~m}$ & $4.4 \mathrm{E}+02$ \\
Se- 83 & $3.0 \mathrm{E}+01$ \\
Sn-110 & $8.9 \mathrm{E}+00$ \\
Sn-111 & $6.8 \mathrm{E}+01$ \\
Sn-113 & $5.0 \mathrm{E}-02$ \\
Sn-117m & $1.9 \mathrm{E}-01$ \\
Sn-119m & $1.7 \mathrm{E}-01$
\end{tabular}

Table 2. Trigger Values

\begin{tabular}{lc}
\hline \hline & $\begin{array}{c}\text { Trigger } \\
\text { Value }\end{array}$ \\
Radionuclide & Ci \\
\hline Sn-121 & $1.3 \mathrm{E}+01$ \\
Sn-123 & $3.4 \mathrm{E}-02$ \\
Sn-123m & $2.9 \mathrm{E}+02$ \\
Sn-125 & $5.5 \mathrm{E}-02$ \\
Sn-127 & $6.8 \mathrm{E}+00$ \\
Sn-128 & $1.9 \mathrm{E}+01$ \\
Xe-120 & $3.4 \mathrm{E}+01$ \\
Xe-121 & $3.3 \mathrm{E}+01$ \\
Xe-122 & $3.7 \mathrm{E}+00$ \\
Xe-123 & $6.4 \mathrm{E}+01$ \\
Xe-125 & $2.0 \mathrm{E}-01$ \\
Xe-127 & $3.5 \mathrm{E}+02$ \\
Xe-129m & $4.0 \mathrm{E}+03$ \\
Xe-131m & $1.1 \mathrm{E}+04$ \\
Xe-133 & $2.7 \mathrm{E}+03$ \\
Xe-133m & $3.1 \mathrm{E}+03$ \\
Xe-135 & $3.9 \mathrm{E}+02$ \\
Xe-135m & $3.1 \mathrm{E}+02$ \\
Xe-138 & $5.3 \mathrm{E}+01$
\end{tabular}




\section{REFERENCES}

Chandler, Timothy E., Recommended Radiological and Chemical WAC and Permit Limits for Low-Curie Salt Operations $(0.2 \mathrm{Ci} / \mathrm{gal})$ at the Saltstone Facility (not including Tank 48H), memo to Dennis G. Thompson, WSP-SSF-2004-00030, Revision 0. Westinghouse Savannah River Company, Aiken, SC. August 12, 2004.

Cook, James R., Kocher, David C., McDowell-Boyer, Laura and Wilhite, Elmer L. Special Analysis: Reevaluation of the Inadvertent Intruder, Groundwater, Air and Radon Analyses for the Saltstone Disposal Facility, WSRC-TR-2002-00456, Revision 0. Westinghouse Savannah River Company, Aiken, SC. October 23, 2002.

Cook, James R., 20o4. Special Analysis: Radionuclide Screening Analysis for E-Area, WSRC-TR-2004-00294. Westinghouse Savannah River Company, Aiken, SC. June 1, 2004.

Martin Marietta Energy Systems, EG\&G Idaho, Westinghouse Hanford Company and Westinghouse Savannah River Company, 1992. Radiological Performance Assessment for the Z-Area Saltstone Disposal Facility, WSRC-RP-92-1360. Westinghouse Savannah River Company, Aiken, SC. December 18, 1992.

NCRP, 1996. National Council on Radiation Protection and Measurements, Screening Models for Releases of Radionuclides to Atmosphere, Surface Water, and Ground, NCRP Report No. 123, Volumes I and II, January, 1996.

USDOE, 1999. "Low Level Waste Requirements", Chapter III in Iradioactive Waste Management Manual, USDOE Order M 435.1-1, U. S. Department of Energy, Washington DC, July 9, 1999. 


\section{DESIGN CHECK PACKAGE}


THIS PAGE INTENTIONALLY LEFT BLANK 


\section{DESIGN CHECK INSTRUCTIONS FOR ATMOSPHERIC PATHWAY SCREENING REPORT}

Perform a design check for Atmospheric Pathway Screening Analysis for Saltstone Disposal Facility Vault 4 following the general guidance provided in WSRC-IM-2002-00011.

Specific instructions are as follows:

Check that the Screening Factors have been accurately transcribed from the NCRP Report 123.

Check that the Screening Dose calculations have been computed properly following the Screening Level I procedure on page 30 of NCRP Report 123, Volume II.

Check that the Trigger Values have been computed properly.

Verify that the contents of the spreadsheet used to make the calculations have been accurately transcribed into the tables in the report.

\section{DESIGN CHECK REPORT}

Greater than 10 percent of the Screening factors were checked and several transcription errors in the exponential factors were noted. These changes were addressed and all transcribed data was rechecked prior to proceeding with the design check.

The Screening Dose calculations were computed independently and greater than 10 percent of these calculated values were compared with the Screening Dose values in the report. Within round off error the values were in agreement.

10 percent of the reported Trigger Values were compared with independently calculated values. Within round off error the values were in agreement.

The aforementioned spot checks with independently calculated values provide a verification that the data have been accurately transcribed into the tables in the report. 
THIS PAGE INTENTIONALLY LEFT BLANK 